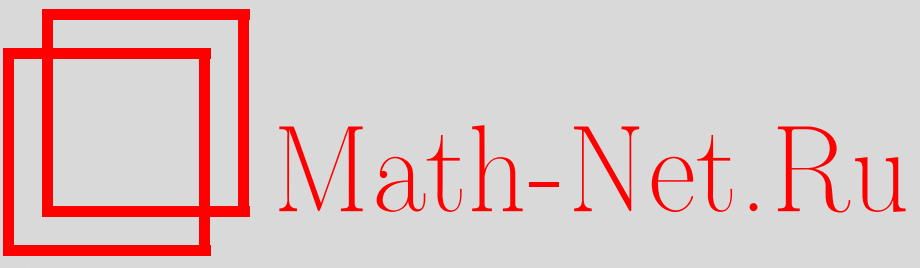

Yu. L. Trakhinin, Local existence of contact discontinuities in relativistic magnetohydrodynamics, Mat. Tr., 2019, Volume 22, Number 2, 175-209

DOI: https://doi.org/10.33048/mattrudy.2019.22.210

Use of the all-Russian mathematical portal Math-Net.Ru implies that you have read and agreed to these terms of use

http://www.mathnet.ru/eng/agreement

Download details:

IP : 52.87 .193 .239

April 26, 2023, 14:57:28 


\title{
ЛОКАЛЬНОЕ СУЩЕСТВОВАНИЕ КОНТАКТНЫХ РАЗРЫВОВ В РЕЛЯТИВИСТСКОЙ МАГНИТНОЙ ГИДРОДИНАМИКЕ
}

\author{
Ю. Л. Трахинин
}

\begin{abstract}
Рассматривается задача со свободной границей, являющейся контактным разрывом для системы уравнений релятивистской магнитной гидродинамики. Поверхность контактного разрыва является характеристикой этой системы и движется со скоростью частиц среды. Функции давления, скорости и магнитного поля непрерывны в точках поверхности, в то время как плотность, энтропия и температура могут иметь произвольный скачок на разрыве. Для двумерного случая доказана локальная по времени теорема существования и единственности в пространствах Соболева решения задачи со свободной границей при условии, что в начальный момент времени в каждой точке разрыва выполнено условие Рэлея - Тейлора на знак скачка производной давления по направлению нормали к разрыву.
\end{abstract}

Ключевые слова и фразы: релятивистская магнитная гидродинамика, задача со свободной границей, контактный разрыв, локальная теорема существования и единственности.

\section{§1. Постановка задачи со свободной границей}

Рассматриваются уравнения релятивистской магнитной гидродинамики (РМГД), описывающие течение невязкого идеально проводящего релятивистского газа (в частности, плазмы) в магнитном поле [7; 16]:

$$
\nabla_{\alpha}\left(\rho u^{\alpha}\right)=0, \quad \nabla_{\alpha} T^{\alpha \beta}=0, \quad \nabla_{\alpha}\left(u^{\alpha} b^{\beta}-u^{\beta} b^{\alpha}\right)=0,
$$

где $\nabla_{\alpha}-$ ковариантная производная относительно лоренцевой метрики $g=\operatorname{diag}(-1,1,1,1)$ в пространстве-времени с компонентами $g_{\alpha \beta}$ (мы ограничиваемся случаем специальной теории относительности), $\rho$ - собственная плотность газа, $u^{\alpha}-$ компоненты 4-скорости,

$$
T^{\alpha \beta}=\left(\rho h+B^{2}\right) u^{\alpha} u^{\beta}+q g^{\alpha \beta}-b^{\alpha} b^{\beta},
$$

$h=1+e+(p / \rho)-$ релятивистская удельная энтальпия, $p-$ давление, $e=e(\rho, S)$ - удельная внутренняя энергия, $S-$ удельная энтропия,

(C) Ю. Л. Трахинин; 2019 
$B^{2}=b^{\alpha} b_{\alpha}-$ магнитное давление (здесь и далее по повторяющимся индексам, обозначенным греческими буквами, берется суммирование от 0 до 3), $b^{\alpha}$ - компоненты 4-вектора магнитного поля и $q=p+\frac{1}{2} B^{2}-$ полное давление. Компоненты 4-векторов удовлетворяют соотношениям $u^{\alpha} u_{\alpha}=-1$ и $u^{\alpha} b_{\alpha}=0$. Будем также предполагать, что выбрана естественная система единиц, в которой скорость света $c=1$.

Пусть $\left(x^{0}, x\right)$ - инерциальная система координат, где $t=x^{0}-$ время, а $x=\left(x_{1}, x_{2}, x_{3}\right)$ - пространственные координаты. Тогда

$$
\begin{gathered}
u^{0}=-u_{0}=\Gamma, \quad u^{i}=u_{i}=\Gamma v_{i}, \quad i=\overline{1,3}, \quad u=\left(u_{1}, u_{2}, u_{3}\right)=\Gamma v, \\
\Gamma^{2}=1+|u|^{2}, \quad b^{0}=-b_{0}=(u \cdot H), \quad b^{i}=b_{i}=\frac{H_{i}}{\Gamma}+(u \cdot H) v_{i}, \\
b=\left(b_{1}, b_{2}, b_{3}\right), \quad B^{2}=|b|^{2}-b_{0}^{2}=\frac{|H|^{2}}{\Gamma^{2}}+(v \cdot H)^{2}>0,
\end{gathered}
$$

где $\Gamma=\left(1-|v|^{2}\right)^{-1 / 2}-$ множитель Лоренца, $v=\left(v_{1}, v_{2}, v_{3}\right)-3$-скорость, a $H=\left(H_{1}, H_{2}, H_{3}\right)-3$-вектор магнитного поля. Здесь и далее при записи в строке некоторого вектор-столбца $a$ в виде $a=\left(a_{1}, a_{2}, a_{3}\right)$ мы для краткости опускаем значок транспонирования, но в то же время $a^{\top}$, фигурирующее внутри некоторой формулы, будет обозначать соответствующую вектор-строку. В терминах 3-векторов уравнения РМГД (1) могут быть переписаны в виде следующей системы законов сохранения:

$$
\begin{aligned}
& \partial_{t}(\rho \Gamma)+\operatorname{div}(\rho u)=0, \\
& \partial_{t}\left(\rho h \Gamma u+|H|^{2} v-(v \cdot H) H\right)+\operatorname{div}\left(\left(\rho h+B^{2}\right) u \otimes u-b \otimes b\right)+\nabla q=0, \\
& \partial_{t}\left(\rho h \Gamma^{2}+|H|^{2}-q\right)+\operatorname{div}\left(\rho h \Gamma u+|H|^{2} v-(v \cdot H) H\right)=0, \\
& \partial_{t} H-\nabla \times(v \times H)=0,
\end{aligned}
$$

где $\partial_{t}=\partial / \partial t, \nabla=\left(\partial_{1}, \partial_{2}, \partial_{3}\right)$ и $\partial_{i}=\partial / \partial x_{i}$. Более того, первое из уравнений Максвелла из системы (1) записывается в виде

$$
\operatorname{div} H=0
$$

и является дивергентным ограничением на начальные данные $U(0, x)=$ $U_{0}(x)$, где в качестве неизвестного мы взяли $U=(p, u, H, S)$.

Используя (6) и дополнительный закон сохранения энтропии

$$
\partial_{t}(\rho \Gamma S)+\operatorname{div}(\rho S u)=0
$$

который выполняется на гладких решениях уравнений (2)-(5), и следуя процедуре симметризации Годунова [4], мы можем симметризовать систему законов сохранения (2)-(5) в терминах вектора множителей Лагранжа 
$Q=Q(U)$. Это было независимо сделано в работах [8; 19], однако конкретный вид симметрических матриц там не был найден. Более того, если мы имеем дело с начально-краевой задачей, а тем более с задачей со свободной границей, то очень неудобно и даже часто технически невозможно работать в терминах вектора $Q$.

С одной стороны, от вектора $Q$ мы можем вернуться к вектору исходных (физических) неизвестных $U$ с сохранением свойства симметричности (см. [2]). Но, с другой стороны, для РМГД нахождение матриц для симметрического вида в терминах $Q$ и возврат к $U$ связаны с невообразимыми (или даже нереализуемыми на практике) вычислениями. Другой возможный способ симметризации системы (2)-(5) мог бы быть основан на переписывании этой системы в неконсервативном симметрическом виде для вектора $U$ по аналогии с тем, как это было сделано в [26] для релятивистской газовой динамики. Но и этот способ для громоздкой системы РМГД трудно реализуем на практике.

Указанная принципиальная трудность преодолена в [11] с помощью применения преобразования Лоренца, а именно, с учетом (6) для состояния покоя $v=0$ мы можем записать уравнения $(2),(3),(5)$ и (7) в неконсервативном и уже симметрическом виде. После этого мы должны надлежащим образом применить преобразование Лоренца к полученной симметрической системе, что даст соответствующий симметрический вид в лабораторной системе отсчета. Отсылая читателя к работе [11], мы здесь просто выпишем полученный там симметрический вид уравнений РМГД:

$$
A_{0}(U) \partial_{t} U+\sum_{j=1}^{3} A_{j}(U) \partial_{j} U=0
$$

где

$$
\begin{gathered}
A_{0}=\left(\begin{array}{cccc}
\frac{\Gamma}{\rho a^{2}} & v^{\top} & 0 & 0 \\
v & \mathcal{A} & 0 & 0 \\
0 & 0 & \mathcal{M} & 0 \\
0 & 0 & 0 & 1
\end{array}\right), \quad A_{j}=\left(\begin{array}{cccc}
\frac{u_{j}}{\rho a^{2}} & e_{j}^{\top} & 0 & 0 \\
e_{j} & \mathcal{A}_{j} & \mathcal{N}_{j}^{\top} & 0 \\
0 & \mathcal{N}_{j} & v_{j} \mathcal{M} & 0 \\
0 & 0 & 0 & v_{j}
\end{array}\right), \\
a^{2}=p_{\rho}(\rho, S), \quad e_{j}=\left(\delta_{1 j}, \delta_{2 j}, \delta_{3 j}\right), \\
\mathcal{A}=\left(\rho h \Gamma+\frac{|H|^{2}}{\Gamma}\right) I-\left(\rho h \Gamma+\frac{|H|^{2}+B^{2}}{\Gamma}\right) v \otimes v \\
\mathcal{M}=\frac{1}{\Gamma}(I+u \otimes u), \quad \mathcal{N}_{j}=\frac{1}{\Gamma} b \otimes e_{j}-\frac{v_{j}}{\Gamma} b \otimes v-\frac{H_{j}}{\Gamma^{2}} I
\end{gathered}
$$




$$
\begin{aligned}
\mathcal{A}_{j}=v_{j} & \left\{\left(\rho h \Gamma+\frac{|H|^{2}}{\Gamma}\right) I-\left(\rho h \Gamma+\frac{|H|^{2}-B^{2}}{\Gamma}\right) v \otimes v-\frac{1}{\Gamma} H \otimes H\right\} \\
& +\frac{H_{j}}{\Gamma}\left\{\frac{1}{\Gamma^{2}}(v \otimes H+H \otimes v)-2(v \cdot H)(I-v \otimes v)\right\} \\
& +\frac{(v \cdot H)}{\Gamma}\left(H \otimes e_{j}+e_{j} \otimes H\right)-\frac{B^{2}}{\Gamma}\left(v \otimes e_{j}+e_{j} \otimes v\right),
\end{aligned}
$$

a $I$ - единичная матрица. Очевидно, что матрицы $A_{\alpha}(\alpha=\overline{0,3})$ симметрические. Заметим, что последнее уравнение в (8) является просто неконсервативной записью $\mathrm{d} S / \mathrm{d} t=0$ закона сохранения $(7)$, где $\mathrm{d} / \mathrm{d} t=\partial_{t}+(v \cdot \nabla)$.

Как известно [7], естественные физические ограничения, гарантирующие гиперболичность системы РМГД не зависят от магнитного поля и совпадают с соответствующими ограничениями в релятивистской газовой динамике [26]. В нашем случае прямыми вычислениями можно показать, что условие гиперболичности $A_{0}>0$ равносильно выполнению неравенств

$$
\rho>0, \quad p_{\rho}>0, \quad 0<c_{s}^{2}<1
$$

(по умолчанию, конечно, также предполагается, что $|v|<1$ ), где $c_{s}-$ релятивистская скорость звука: $c_{s}^{2}=a^{2} / h=p_{\rho} / h$. Последнее неравенство в (9) называется условием релятивистской причинности.

Мы будем рассматривать уравнения РМГД (1) (или (8)) при $t \in[0, T]$ во всем трехмерном пространстве $\mathbb{R}^{3}$. Пусть

$$
\Gamma(t)=\left\{x_{1}-\varphi\left(t, x^{\prime}\right)=0\right\}
$$

- некоторая гладкая гиперповерхность в $[0, T] \times \mathbb{R}^{3}$, где $x^{\prime}=\left(x_{2}, x_{3}\right)$. Предположим, что $\Gamma(t)$ является поверхностью сильного разрыва для системы законов сохранения (1); т. е. нас интересуют решения уравнений (1), которые являются гладкими в

$$
\Omega^{ \pm}(t)=\left\{ \pm\left(x_{1}-\varphi\left(t, x^{\prime}\right)\right)>0\right\}
$$

в то время как компоненты $U$ могут иметь скачок на разрыве Г. Как известно, для того чтобы соответствующее кусочно-гладкое решение законов сохранения (1) во всем $\mathbb{R}^{3}=\Omega^{+}(t) \cup \Omega^{-}(t)$ было слабым решением системы (1), необходимо выполнение стандартных соотношений на скачки (типа условий Ренкина - Гюгонио) в каждой точке $\Gamma(t)$ :

$$
\begin{aligned}
& {[\mathfrak{j}] }=0, \\
& \mathfrak{j}\left[\left(h \Gamma+\frac{|H|^{2}}{\rho \Gamma}\right) v_{n}+\frac{(v \cdot H)}{\rho \Gamma} H_{n}\right]-H_{n}\left[\frac{H_{n}}{\Gamma^{2}}+(v \cdot H) v_{n}\right]+[q]=0,
\end{aligned}
$$




$$
\begin{gathered}
\mathfrak{j}\left[\left(h \Gamma+\frac{|H|^{2}}{\rho \Gamma}\right) v_{\tau}+\frac{(v \cdot H)}{\rho \Gamma} H_{\tau}\right]-H_{n}\left[\frac{H_{\tau}}{\Gamma^{2}}+(v \cdot H) v_{\tau}\right]=0, \\
\mathfrak{j}\left[\frac{H_{\tau}}{\rho \Gamma}\right]-H_{n}\left[v_{\tau}\right]=0, \\
\mathfrak{j}\left[h \Gamma+|H|^{2}+\frac{q}{\rho \Gamma}\right]+H_{n}[v \cdot H]+\left[v_{n} q\right]=0,
\end{gathered}
$$

где $[g]=g_{\mid \Gamma}^{+}-g_{\mid \Gamma}^{-}$обозначает скачок некоторой величины $g$, а $g^{ \pm}:=g$ в $\Omega^{ \pm}(t)$,

$$
\begin{gathered}
\mathfrak{j}^{ \pm}=\rho^{ \pm} \Gamma^{ \pm}\left(v_{n}^{ \pm}-\sigma\right), \quad v_{n}^{ \pm}=v^{ \pm} \cdot n, \quad \mathfrak{j}:=\left.\mathfrak{j}^{ \pm}\right|_{\Gamma}, \\
n=\frac{1}{\sqrt{1+\left(\partial_{2} \varphi\right)^{2}+\left(\partial_{3} \varphi\right)^{2}}}\left(1,-\partial_{2} \varphi,-\partial_{3} \varphi\right), \quad \sigma=\frac{\partial_{t} \varphi}{\sqrt{1+\left(\partial_{2} \varphi\right)^{2}+\left(\partial_{3} \varphi\right)^{2}}}, \\
H_{n}^{ \pm}=H^{ \pm} \cdot n, \quad H_{n}:=\left.H_{n}^{ \pm}\right|_{\Gamma}, \\
v_{\tau}^{ \pm}=\left(v_{\tau_{1}}^{ \pm}, v_{\tau_{2}}^{ \pm}\right), \quad v_{\tau_{i}}^{ \pm}=\left(v^{ \pm} \cdot \tau_{i}\right), \quad \tau_{1}=\left(\partial_{2} \varphi, 1,0\right), \quad \tau_{2}=\left(\partial_{3} \varphi, 0,1\right), \\
H_{\tau}^{ \pm}=\left(H_{\tau_{1}}^{ \pm}, H_{\tau_{2}}^{ \pm}\right), \quad H_{\tau_{i}}^{ \pm}=\left(H^{ \pm} \cdot \tau_{i}\right) .
\end{gathered}
$$

Как и в нерелятивистской МГД $[2 ; 5 ; 17 ; 18]$, контактным разрывом будем называть такой разрыв, что поток массы через него равен нулю, а магнитное поле в точках разрыва нигде ему не параллельно: $\mathfrak{j}=0$ и $H_{n} \neq 0$. Тогда $\sigma=\left.v_{n}^{ \pm}\right|_{\Gamma}$, т. е. $\left[v_{n}\right]=0$, а из (14) выводим $\left[v_{\tau}\right]=0$. Так как $[v]=0$, из $(12)$ получаем

$$
\left(1-\sigma^{2}\right)\left[H_{\tau}\right]=0 .
$$

Поскольку скорость разрыва $\sigma$ меньше скорости света, то $1-\sigma^{2}>0$ и из $(16)$ имеем $\left[H_{\tau}\right]=0$. Таким образом, с учетом $(13)$ получаем $[H]=0$. Далее, из (11) следует $[p]=0$, а (15) выполняется автоматически; т. е., как и в нерелятивистском случае [2; 18], мы получаем следующие граничные условия на контактном разрыве:

$$
[p]=0, \quad[v]=0, \quad[H]=0, \quad \partial_{t} \varphi=\left.v_{N}^{+}\right|_{\Gamma},
$$

где $v_{N}^{+}=v_{1}^{+}-v_{2}^{+} \partial_{2} \varphi-v_{3}^{+} \partial_{3} \varphi$. Итак, контактный разрыв движется со скоростью частиц среды и в точках его поверхности непрерывны давление, скорость и магнитного поле. В то же время плотность и энтропия (а также температура $\left.T=e_{S}(\rho, S)\right)$ могут иметь произвольный скачок на разрыве. Здесь и далее нам будет удобнее записывать граничные условия в терминах вектора $v=u / \Gamma=u / \sqrt{1+|u|^{2}}$ (понятно, что условия $[v]=0$ и $[u]=0$ равносильны). 
Как отмечается в [12], граничные условия вида (17) наиболее типичны в солнечном ветре и астрофизической плазме (т. е. в плазме за границей солнечной системы). Контактные разрывы наблюдаются за астрофизическими ударными волнами, ограничивающими остатки сверхновой, или, например, появляются в результате взаимодействия множественных ударных волн, порождаемых быстрыми корональными выбросами массы. Если характерные скорости сравнимы со скоростью света, что возможно, например, в астрофизической плазме, то необходимо рассматривать контактные разрывы в рамках релятивистских моделей, в частности, в рамках модели РМГД.

С математической точки зрения задача со свободной границей для контактного разрыва является задачей поиска решений систем

$$
A_{0}\left(U^{ \pm}\right) \partial_{t} U^{ \pm}+\sum_{j=1}^{3} A_{j}\left(U^{ \pm}\right) \partial_{j} U^{ \pm}=0 \quad \text { при } x \in \Omega^{ \pm}(t)
$$

$($ см. $(8))$, удовлетворяющих граничным условиям $(17)$ на $\Gamma(t)$ и начальным данным

$$
U^{ \pm}(0, x)=U_{0}^{ \pm}(x), \quad x \in \Omega^{ \pm}(0), \quad \varphi\left(0, x^{\prime}\right)=\varphi_{0}\left(x^{\prime}\right), \quad x^{\prime} \in \mathbb{R}^{2},
$$

при $t=0$. Нашей главной целью является нахождение условий на начальные данные (19), гарантирующие существование и единственность гладкого решения $\left(U^{+}, U^{-}, \varphi\right)$ задачи со свободной границей (17)-(19) на некотором интервале времени $[0, T]$. Эти условия будут дополнительными к условию гиперболичности (9) и требованию $H_{n} \neq 0$.

Забегая вперед, заметим, что для доказательства теоремы существования и единственности гладкого решения задачи (17)-(19) нам необходимо просто сделать небольшую ревизию нескольких ключевых моментов доказательства аналогичной теоремы для контактных разрывов в нерелятивистской МГД [17; 18]. В [18] удалось доказать теорему существования и единственности только для двумерного варианта задачи, точнее сказать, для двумерного плоскостного течения. Следуя [17; 18], для РМГД мы также будем рассматривать такое течение. Это означает, что течение $x_{3}$-инвариантно, т. е. $U=U\left(t, x_{1}, x_{2}\right)$, а третьи компоненты скорости и магнитного поля равны нулю: $v_{3}=H_{3}=0$. Для нерелятивистской МГД ограничение $\left.v_{3}\right|_{t=0}=\left.H_{3}\right|_{t=0}=0$ на начальные данные гарантирует то, что двумерное течение является плоскостным. Нетрудно доказать, что то же самое имеет место и для РМГД.

В самом деле, известно [3; 15], что для гладких начальных данных симметрическая гиперболическая система имеет единственное гладкое решение на малом отрезке времени $[0, T]$. Тогда естественно предполагать, 
что система (1) (или (8)) имеет гладкие решения для $t \in[0, T]$. Опуская простые технические вычисления, заметим, что четвертое и последнее уравнения системы $(1)$ для $U=U\left(t, x_{1}, x_{2}\right)$ можно переписать в виде симметрической гиперболической системы

$$
\left(\begin{array}{cc}
\Gamma^{2}\left(\rho h \Gamma^{2}+|H|^{2}\right) & 0 \\
0 & 1
\end{array}\right) \partial_{t} W+\sum_{j=1}^{2}\left(\begin{array}{cc}
a_{1} v_{j}+a_{2} H_{j} & -H_{j} \\
-H_{j} & v_{j}
\end{array}\right) \partial_{j} W+B W=0
$$

для $W=\left(v_{3}, H_{3}\right)$, где конкретный вид функций $a_{1}=a_{1}(U)$ и $a_{2}=a_{2}(U)$, а также матрицы $B=B\left(U, U_{t}, \partial_{1} U, \partial_{2} U\right)$ не имеет значения. При выполнении условия гиперболичности $(9)$ матрица перед $\partial_{t} W$ положительно определенная. Тогда из априорной $L^{2}$-оценки для решений $W$ этой системы и предположения $\left.W\right|_{t=0}=0$ следует $W=0$ для $t \in[0, T]$. Таким образом, в РМГД правомерно рассматривать двумерные плоскостные течения.

Рассматривая далее задачу (17)-(19) для двумерного плоскостного течения, мы, не нарушая общности, будем считать, что пространственные переменные, скорость и магнитное поле имеют только две компоненты: $x=\left(x_{1}, x_{2}\right) \in \mathbb{R}^{2}, v^{ \pm}=\left(v_{1}^{ \pm}, v_{2}^{ \pm}\right) \in \mathbb{R}^{2}$ и $H^{ \pm}=\left(H_{1}^{ \pm}, H_{2}^{ \pm}\right) \in \mathbb{R}^{2}$. Тогда

$$
v_{N}^{ \pm}=v_{1}^{ \pm}-v_{2}^{ \pm} \partial_{2} \varphi, \quad H_{N}^{ \pm}=H_{1}^{ \pm}-H_{2}^{ \pm} \partial_{2} \varphi, \quad v_{\tau}^{ \pm}=v_{1}^{ \pm} \partial_{2} \varphi+v_{2}^{ \pm}
$$

и т. д.

Заметим, что для контактного разрыва в нерелятивистской МГД результаты работ [17] (для линеаризованной задачи) и [18] (для исходной нелинейной задачи) получены для таких уравнений состояния газа, что из $[p]=0$ следует $\left[\rho p_{\rho}\right]=0$. Это, в частности, выполнено для уравнения состояния политропного газа

$$
\rho(p, S)=A p^{\frac{1}{\gamma}} e^{-\frac{S}{\gamma}}, \quad A>0, \quad \gamma>1 .
$$

На самом деле, в $[17 ; 18]$ для определенности рассматривался именно политропный газ. Следуя [17; 18], мы будем предполагать, что релятивистский газ политропный. Заметим, что в астрофизике для релятивистского (и нерелятивистского) газа делается это предположение. Для политропного газа первые два неравенства в условии гиперболичности (9) сводятся к требованию

$$
p>0 .
$$

Выполнение (21) гарантирует также справедливость неравенства $c_{s}^{2}>0$. Условие же релятивистской причинности $c_{s}^{2}<1$ сводится к неравенству

$$
1+\frac{\gamma(2-\gamma) p}{(\gamma-1) \rho}>0
$$


При условии (21) последнее неравенство заведомо выполняется для $\gamma \leq 2$ (по умолчанию предполагается, что $\gamma>1$, см. (20)). Совсем необязательно предполагать, что показатель адиабаты $\gamma$ не больше 2 , но мы для простоты ссылок на работы $[17 ; 18]$ будем далее по умолчанию считать, что $\gamma \leq 2$. Тогда, как и в [17; 18], условие гиперболичности сводится к единственному неравенству (21). На самом деле, в астрофизике обычно предполагается, что газ одноатомный. Это означает, что $\gamma=5 / 3$ для нерелятивистского газа и $\gamma=4 / 3$ для релятивистского; т. е. в этом случае условие $\gamma \leq 2$ заведомо выполняется.

\section{§2. Приведенная нелинейная задача в фиксированной области}

Для того чтобы свести нашу задачу со свободной границей (для двумерного случая) к задаче в фиксированной области, мы «распрямляем» кривую Г, используя такую же простейшую замену независимых переменных, как и в $[25 ; 26]$; т. е. неизвестные $U^{+}$и $U^{-}$, будучи гладкими в $\Omega^{ \pm}(t)$, заменяются вектор-функциями

$$
\widetilde{U}^{ \pm}(t, x):=U^{ \pm}\left(t, \Phi^{ \pm}(t, x), x_{2}\right),
$$

которые являются гладкими в полуплоскости $\mathbb{R}_{+}^{2}=\left\{x_{1}>0, x_{2} \in \mathbb{R}\right\}$, где

$$
\Phi^{ \pm}(t, x):= \pm x_{1}+\Psi^{ \pm}(t, x), \quad \Psi^{ \pm}(t, x):=\chi\left( \pm x_{1}\right) \varphi\left(t, x_{2}\right),
$$

функция срезки $\chi \in C_{0}^{\infty}(\mathbb{R})$ равна 1 на $[-1,1]$ и $\left\|\chi^{\prime}\right\|_{L_{\infty}(\mathbb{R})}<1 / 2$. Функция срезки используется для того, чтобы избежать предположения о финитности начальных данных в теореме существования. Замена (22) допустима, если $\partial_{1} \Phi^{ \pm} \neq 0$. Последнее гарантировано, т. е. справедливы неравенства $\partial_{1} \Phi^{+}>0$ и $\partial_{1} \Phi^{-}<0$, если для решений задачи выполняется

$$
\|\varphi\|_{L_{\infty}([0, T] \times \mathbb{R})} \leq 1
$$

Это справедливо, если, не нарушая общности, рассматриваем начальные данные, для которых $\left\|\varphi_{0}\right\|_{L_{\infty}(\mathbb{R})} \leq 1 / 2$, а время $T$ существования решений в нашей теореме существования (см. ниже теорему 3.1) достаточно мало́.

Опуская для удобства «волны» над $\widetilde{U}^{ \pm}$, мы приводим задачу (17)-(19) (в двумерном случае) к начально-краевой задаче

$$
\begin{gathered}
A_{0}\left(U^{ \pm}\right) \partial_{t} U^{ \pm}+\widetilde{A}_{1}\left(U^{ \pm}, \Psi^{ \pm}\right) \partial_{1} U^{ \pm}+A_{2}\left(U^{ \pm}\right) \partial_{2} U^{ \pm}=0 \quad \text { в } \quad[0, T] \times \mathbb{R}_{+}^{2}, \\
{[p]=0, \quad[v]=0, \quad[H]=0, \quad \partial_{t} \varphi=v_{N}^{+} \quad \text { на }[0, T] \times\left\{x_{1}=0\right\} \times \mathbb{R}} \\
U_{\mid t=0}^{+}=U_{0}^{+}, \quad U_{\mid t=0}^{-}=U_{0}^{-} \quad \text { в } \quad \mathbb{R}_{+}^{2},\left.\quad \varphi\right|_{t=0}=\varphi_{0} \quad \text { в } \quad \mathbb{R}
\end{gathered}
$$


где

$$
\widetilde{A}_{1}\left(U^{ \pm}, \Psi^{ \pm}\right)=\frac{1}{\partial_{1} \Phi^{ \pm}}\left(A_{1}\left(U^{ \pm}\right)-A_{0}\left(U^{ \pm}\right) \partial_{t} \Psi^{ \pm}-A_{2}\left(U^{ \pm}\right) \partial_{2} \Psi^{ \pm}\right)
$$

$\left(\partial_{1} \Phi^{ \pm}= \pm 1+\partial_{1} \Psi^{ \pm}\right)$и в $(26)$ мы используем обозначение $[g]:=g_{\mid x_{1}=0}^{+}-g_{\mid x_{1}=0}^{-}$ для любой пары величин $g^{+}$и $g^{-}$.

Нас интересуют гладкие решения $\left(U^{+}, U^{-}, \varphi\right)$ задачи $(25)-(27)$, точнее сказать, как и в $[17 ; 18]$, мы собираемся доказать их существование при выполнении условия гиперболичности (21), требований $|v|<1, H_{n} \neq 0$ и условия Рэлея - Тейлора $[\partial p / \partial n]<0$ в начальный момент времени. Иными словами, начальные данные должны будут удовлетворять неравенствам

$$
\begin{aligned}
p^{ \pm} & \geq \bar{p}>0, \\
1-\left|v^{ \pm}\right| & \geq \nu>0, \\
\left|H_{N}^{ \pm}\right|_{x_{1}=0} \mid & \geq \kappa>0, \\
{\left[\partial_{1} p\right] } & \geq \epsilon>0,
\end{aligned}
$$

где $\bar{p}, \nu, \kappa$ и $\epsilon$ - положительные постоянные, $(31)$ - условие Рэлея - Тейлора, записанное для «распрямленного» разрыва (с уравнением $x_{1}=0$ ). Поскольку подвижные области $\Omega^{ \pm}(t)$ сведены к одной и той же полуплоскости $\mathbb{R}_{+}^{2}$ (а не к разным полуплоскостям $\mathbb{R}_{+}^{2}$ и $\mathbb{R}_{-}^{2}$ ), скачок производной по направлению нормали определяется так:

$$
\left[\partial_{1} a\right]:=\partial_{1} a_{\mid x_{1}=0}^{+}+\partial_{1} a_{\mid x_{1}=0}^{-} .
$$

Так как область $\mathbb{R}_{+}^{2}$ неограниченная, то функции из соболевских пространств $H^{s}\left(\mathbb{R}_{+}^{2}\right)$ должны стремиться к нулю на бесконечности. Поэтому, говоря о гладких решениях задачи (25)-(27), мы будем требовать, чтобы не сами $U^{+}$и $U^{-}$, а соответствующие вектор-функции, сдвинутые на некоторые гладкие ограниченные вектор-функции $\bar{U}^{+}$и $\bar{U}-$, принадлежали $H^{s}$. В самом деле, неравенства $(28),(30)$ и (31) не могут быть выполнены в $\mathbb{R}_{+}^{2}$ для $U^{+}$и $U^{-}$, стремящихся к нулю на бесконечности. В качестве сдвигов $\bar{U}^{ \pm}$можно взять магнитостатические равновесия $\bar{U}^{ \pm}(x)$, являющиеся некоторыми гладкими ограниченными решениями стационарной системы РМГД и удовлетворяющие условию $(28)$ во всей полуплоскости $\mathbb{R}_{+}^{2}$ и условиям (30) и (31) на ее границе $x_{1}=0$. Для нерелятивистской МГД если учесть влияние гравитации (что естественно, когда идет речь об условии Рэлея - Тейлора) и ввести в уравнения МГД младшие гравитационные члены, то можно привести примеры достаточно простых равновесий [18], удовлетворяющих условиям (28), (30) и (31), когда контактный разрыв располагается между двумя бесконечно проводимыми твердыми границами (для двумерного случая эти границы являются прямыми $x_{1}= \pm a$, 
$a=$ const, cм. [18]). Аналогичные магнитостатические равновесия можно найти и для РМГД.

Так как нас интересует корректность задачи, а не устойчивость ее решений, младшие (недифференциальные) гравитационные члены не играют в этом смысле никакой роли. Поэтому, как и в [18], для технической простоты мы не будем вводить в уравнения гравитационные члены и делать сдвиг решений на упомянутые магнитостатические равновесия, а просто поставим периодические в тангенциальном направлении граничные условия. Будем считать, что область течения релятивистского газа есть

$$
D=\left\{x \in \mathbb{R}^{2} \mid x_{1} \in \mathbb{R}, x_{2} \in \mathbb{T}\right\}
$$

где $\mathbb{T}-1$-тор (единичная окружность), т. е. значения решений на концах единичного отрезка на оси $x_{2}$ должны совпадать. Тогда

$$
\Gamma(t)=\left\{x \in \mathbb{R} \times \mathbb{T}, x_{1}=\varphi\left(t, x_{2}\right)\right\}, \quad t \in[0, T] .
$$

Далее, как и выше, мы делаем замену переменных (22), которая приводит задачу со свободной границей к задаче в фиксированной области

$$
\Omega=\left\{x_{1}>0, x_{2} \in \mathbb{T}\right\}
$$

с «распрямленным» контактным разрывом

$$
\partial \Omega=\left\{x_{1}=0, x_{2} \in \mathbb{T}\right\} .
$$

Так как $\Omega$ все еще неограниченная область, для удовлетворения условия гиперболичности (28) при $x_{1} \rightarrow+\infty$ мы делаем обычный сдвиг неизвестных на константы:

$$
\breve{U}^{ \pm}=U^{ \pm}-\bar{U}^{ \pm}
$$

где $\bar{U}^{ \pm}=\left(\bar{p}, 0,0, \bar{S}^{ \pm}\right)$, постоянные $\bar{S}^{ \pm}$такие, что $\bar{S}^{+} \neq \bar{S}^{-}$, а $\bar{p}-$ постоянная из (28). После замены неизвестных (33) граничные условия (26) остаются неизменными, в то время как в системах РМГД (25) мы должны сделать сдвиг аргументов $U^{ \pm}$матриц на постоянные векторы $\bar{U}^{ \pm}$. Опуская для удобства бревисы над $\breve{U^{ \pm}}$, получаем следующую начально-краевую задачу в области $[0, T] \times \Omega$ :

$$
\begin{gathered}
\mathbb{L}\left(U^{+}, \Psi^{+}\right)=0, \quad \mathbb{L}\left(U^{-}, \Psi^{-}\right)=0 \quad \text { в }[0, T] \times \Omega, \\
\mathbb{B}\left(U^{+}, U^{-}, \varphi\right)=0 \quad \text { на }[0, T] \times \partial \Omega, \\
U_{\mid t=0}^{+}=U_{0}^{+}, \quad U_{\mid t=0}^{-}=U_{0}^{-} \quad \text { в } \Omega,\left.\quad \varphi\right|_{t=0}=\varphi_{0} \quad \text { на } \partial \Omega,
\end{gathered}
$$

где $\mathbb{L}\left(U^{ \pm}, \Psi^{ \pm}\right)=L\left(U^{ \pm}, \Psi^{ \pm}\right) U^{ \pm}$,

$$
L\left(U^{ \pm}, \Psi^{ \pm}\right)=A_{0}\left(U^{ \pm}+\bar{U}^{ \pm}\right) \partial_{t}+\widetilde{A}_{1}\left(U^{ \pm}+\bar{U}^{ \pm}, \Psi^{ \pm}\right) \partial_{1}+A_{2}\left(U^{ \pm}+\bar{U}^{ \pm}\right) \partial_{2}
$$


и (35) - компактная форма записи граничных условий

$$
[p]=0, \quad[v]=0, \quad\left[H_{\tau}\right]=0, \quad \partial_{t} \varphi-\left.v_{N}^{+}\right|_{x_{1}=0}=0 .
$$

Здесь и далее

$$
H_{\tau}^{ \pm}=H_{1}^{ \pm} \partial_{2} \Psi^{ \pm}+H_{2}^{ \pm}, \quad H_{N}^{ \pm}=H_{1}^{ \pm}-H_{2}^{ \pm} \partial_{2} \Psi^{ \pm}, \quad v_{N}^{ \pm}=v_{1}^{ \pm}-v_{2}^{ \pm} \partial_{2} \Psi^{ \pm}
$$

$\left(\left.v_{N}^{ \pm}\right|_{x_{1}=0}=\left.\left(v_{1}^{ \pm}-v_{2}^{ \pm} \partial_{2} \varphi\right)\right|_{x_{1}=0}\right.$ и т. д.) Более того, в силу (33) условия гиперболичности для систем (34) могут быть записаны, например, как (см. (28))

$$
p^{ \pm}>-\bar{p} / 4
$$

в то время как условия (30) и (31) для новых («сдвинутых») неизвестных остаются неизменными.

Заметим, что условие непрерывности магнитного поля $[H]=0$ эквивалентно $\left[H_{N}\right]=0$ и $\left[H_{\tau}\right]=0$. Так как уравнения Максвелла (5) полностью совпадают с их нерелятивистской версией, следуя [17], мы делаем вывод о том, что условие $\left[H_{N}\right]=0$ не является граничным условием, а должно рассматриваться как ограничение на начальные данные (36) (поэтому оно и не было включено в граничные условия (37)). Также можно показать, что выполнение уравнения (6) с обеих сторон от разрыва в начальный момент времени гарантирует его справедливость при $t>0$. Точнее сказать, имеет место следующее утверждение.

Утверждение 2.1. Пусть начальные данные (36) удовлетворяют

$$
\operatorname{div} \mathfrak{h}^{+}=0, \quad \operatorname{div} \mathfrak{h}^{-}=0
$$

и условию

$$
\left[H_{N}\right]=0
$$

где $\mathfrak{h}^{ \pm}=\left(H_{N}^{ \pm}, H_{2}^{ \pm} \partial_{1} \Phi^{ \pm}\right)$. Если задача (34)-(36) имеет достаточно гладкое решение, то оно удовлетворяет (39) и (40) для всех $t \in[0, T]$.

Хорошо известно (см., например, [2]), что необходимым условием корректности гиперболической задачи типа задачи (34)-(36) является то, что число граничных условий в некоторой точке границы $\left(t^{*}, x_{2}^{*}\right) \in[0, T] \times \partial \Omega$ должно на единицу превосходить число уходящих (с границы $x_{1}=0$ ) характеристик одномерной системы

$$
\mathfrak{A}_{0} \partial_{t}\left(\begin{array}{l}
U^{+} \\
U^{-}
\end{array}\right)+\mathfrak{A}_{1} \partial_{1}\left(\begin{array}{l}
U^{+} \\
U^{-}
\end{array}\right) \text {при } x_{1}>0
$$

у которой коэффициенты матриц

$$
\mathfrak{A}_{0}=\left(\begin{array}{cc}
A_{0}^{+} & 0 \\
0 & A_{0}^{-}
\end{array}\right) \quad \text { и } \quad \mathfrak{A}_{1}=\left(\begin{array}{cc}
\widetilde{A}_{1}^{+} & 0 \\
0 & -\widetilde{A}_{1}^{-}
\end{array}\right)
$$


фиксированы («заморожены») в указанной точке $\left(t^{*}, x_{2}^{*}\right)$, где

$$
\begin{aligned}
A_{0}^{ \pm} & :=\left.A_{0}\left(U^{ \pm}+\bar{U}^{ \pm}\right)\right|_{x_{1}=0} \\
\widetilde{A}_{1}^{ \pm} & := \pm\left.\widetilde{A}_{1}\left(U^{ \pm}+\bar{U}^{ \pm}, \Psi^{ \pm}\right)\right|_{x_{1}=0} \\
& =\left.\left(A_{1}\left(U^{ \pm}+\bar{U}^{ \pm}\right)-A_{0}\left(U^{ \pm}+\bar{U}^{ \pm}\right) \partial_{t} \varphi-A_{2}\left(U^{ \pm}+\bar{U}^{ \pm}\right) \partial_{2} \varphi\right)\right|_{x_{1}=0} .
\end{aligned}
$$

Это условие продиктовано общим правилом постановки краевых задач для одномерных линейных гиперболических систем и тем, что одно из граничных условий (а в нашем случае это последнее условие в (37)) должно рассматриваться как уравнение для нахождения функции $\varphi\left(t, x_{2}\right)$.

Число уходящих характеристик упомянутой системы равно числу положительных собственных чисел матрицы $\left(A_{0}^{+}\right)^{-1} \widetilde{A}_{1}^{+}$плюс число отрицательных собственных чисел матрицы $\left(A_{0}^{-}\right)^{-1} \widetilde{A}_{1}^{-}$. Очевидно, что

$$
\tilde{\lambda}_{i}^{ \pm}=\lambda_{i}^{ \pm}-\partial_{t} \varphi, \quad i=\overline{1,6}
$$

где $\widetilde{A}_{i}^{ \pm}$- собственные числа матриц $\left(A_{0}^{ \pm}\right)^{-1} \widetilde{A}_{1}^{ \pm}$, а $\lambda_{i}^{ \pm}$- собственные числа характеристических матриц $A_{N}^{ \pm}$гиперболических систем $(34)$, вычисленных на векторе нормали $N=\left(1,-\partial_{2} \varphi\right)$, т. е.

$$
A_{N}^{ \pm}=\left.\left(A_{0}\left(U^{ \pm}+\bar{U}^{ \pm}\right)\right)^{-1}\left(A_{1}\left(U^{ \pm}+\bar{U}^{ \pm}\right)-A_{2}\left(U^{ \pm}+\bar{U}^{ \pm}\right) \partial_{2} \varphi\right)\right|_{x_{1}=0} .
$$

Как и в нерелятивистской МГД [2; 5], собственные числа характеристической матрицы в общем трехмерном случае зависят от альфвеновской, медленной магнитозвуковой и быстрой магнитозвуковой скоростей [7; 16]. Однако, как и в нерелятивистской МГД, для двумерного плоскостного случая, который мы рассматриваем, «выпадает» альфвеновская скорость, т. е. собственные числа определяются только через медленные магнитозвуковые и быстрые магнитозвуковые скорости, а именно, собственные числа $\lambda_{i}^{ \pm}$,

$$
\lambda_{1}^{-} \leq \cdots \leq \lambda_{6}^{-}, \quad \lambda_{1}^{+} \leq \cdots \leq \lambda_{6}^{+}
$$

имеют вид

$$
\begin{aligned}
& \lambda_{1}^{ \pm}=\left.v_{N}^{ \pm}\right|_{x_{1}=0}-c_{f_{-}}^{ \pm}, \quad \lambda_{2}^{ \pm}=\left.v_{N}^{ \pm}\right|_{x_{1}=0}-c_{s_{-}}^{ \pm}, \\
& \lambda_{3}^{ \pm}=\lambda_{4}^{ \pm}=\left.v_{N}^{ \pm}\right|_{x_{1}=0}, \quad \lambda_{5}^{ \pm}=\left.v_{N}^{ \pm}\right|_{x_{1}=0}+c_{s_{+}}^{ \pm}, \quad \lambda_{6}^{ \pm}=\left.v_{N}^{ \pm}\right|_{x_{1}=0}+c_{f_{+}}^{ \pm},
\end{aligned}
$$

где

$$
c_{s_{ \pm}}^{ \pm}=c_{s_{ \pm}}\left(U_{\mid x_{1}=0}^{ \pm}, \varphi\right), \quad c_{f_{ \pm}}^{ \pm}=c_{f_{ \pm}}\left(U_{\mid x_{1}=0}^{ \pm}, \varphi\right),
$$

а $c_{s_{ \pm}} \geq 0$ и $c_{f_{ \pm}} \geq 0$ - соответственно медленные магнитозвуковые и быстрые магнитозвуковые скорости $[7 ; 16]$ (заметим, что в нерелятивистской МГД $c_{s_{-}}=c_{s_{+}}$и $c_{f_{-}}=c_{f_{+}}$; см. $\left.[2 ; 5]\right)$. 
В отличие от нерелятивистского случая, для медленных и быстрых магнитозвуковых скоростей нет «обозримых» явных формул, так как $c_{s_{-}}$, $c_{s_{+}}, c_{f_{-}}$и $c_{f_{+}}$являются корнями полинома четвертой степени $[7 ; 16]$. Однако для нас важно только то, что свободный член этого полинома равен $H_{N} / \Gamma^{3}$ (см. [9]). В силу предположения (30) свободный член на границе не равен нулю, т. е. полином не имеет нулевых корней. Таким образом, все скорости $c_{s_{ \pm}}^{ \pm}$и $c_{f_{ \pm}}^{ \pm}$строго положительные. Тогда из (42) и (43) ввиду последнего условия в (37) следует, что

$$
\widetilde{A}_{1}^{ \pm}=-c_{f_{-}}^{ \pm}, \quad \widetilde{A}_{2}^{ \pm}=-c_{s_{-}}^{ \pm}, \quad \widetilde{A}_{3}^{ \pm}=\widetilde{A}_{4}^{ \pm}=0, \quad \widetilde{A}_{5}^{ \pm}=c_{a_{+}}^{ \pm}, \quad \widetilde{A}_{6}^{ \pm}=c_{f_{+}}^{ \pm} ;
$$

т. е. матрица $\left(\mathfrak{A}_{0}\right)^{-1} \mathfrak{A}_{1}$ в каждой точке границы $x_{1}=0$ имеет четыре положительных собственных значения. Это означает, что системы (34) требуют пять граничных условий, что совпадает с числом условий в (35). Так как граничная матрица $\mathfrak{A}_{1}$ имеет нулевые собственные числа, контактный разрыв является характеристическим. Также важно то, что ранг этой матрицы постоянен (равен восьми).

Ключевым моментом при выводе априорных оценок решения линеаризованной задачи для контактного разрыва в нерелятивистской МГД [17; 18] было предположение, что основное состояние, относительно которого проводится линеаризация, удовлетворяет условию

$$
\left[\partial_{1} v\right]=0
$$

(напомним, что скачок «нормальной» производной определяется в (32)). Важно то, что это условие выполняется для решений исходной нелинейной задачи. Поэтому предположение (45) является естественным и может быть оценена вызванная им дополнительная ошибка итерационной схемы Нэша - Мозера, с помощью которой в [18] доказывалось существование решений нелинейной задачи. В этой связи нам важно доказать, что решения задачи (34)-(36) для контактного разрыва в РМГД также удовлетворяют условию (45) на скачок «нормальной» производной скорости. Более того, можно показать, что эти решения также удовлетворяют условию

$$
\left[\partial_{1} H_{N}\right]=0 .
$$

Утверждение 2.2. Пусть задача (34)-(36) (с начальными данными, удовлетворяющими (39) и (40)) имеет достаточно гладкое решение, для которого справедливы условия (30) и (38). Тогда это решение удовлетворяет равенствам (45) и (46).

Доказательство. Первое уравнение системы (8) записываются так (напомним, что мы рассматриваем политропный газ):

$$
\frac{\Gamma}{\gamma p} \frac{\mathrm{d} p}{\mathrm{~d} t}+v \cdot \partial_{t} u+\operatorname{div} u=0
$$


Тогда после «распрямления» разрыва и замены неизвестных (33) это уравнение дает первые уравнения систем (34)

$$
\begin{aligned}
& \frac{\Gamma^{ \pm}}{\gamma\left(\bar{p}+p^{ \pm}\right)} \partial_{t} p^{ \pm}+v^{ \pm} \cdot \partial_{t} u^{ \pm} \\
& \quad+\frac{1}{\partial_{1} \Phi^{ \pm}}\left\{\frac{\Gamma^{ \pm}}{\gamma\left(\bar{p}+p^{ \pm}\right)}\left(w^{ \pm} \cdot \nabla p^{ \pm}\right)-\partial_{t} \Psi^{ \pm}\left(v^{ \pm} \cdot \partial_{1} u^{ \pm}\right)+\operatorname{div} \widetilde{u}^{ \pm}\right\}=0
\end{aligned}
$$

где

$$
w^{ \pm}=\left(v_{N}^{ \pm}-\partial_{t} \Psi^{ \pm}, v_{2}^{ \pm} \partial_{1} \Phi^{ \pm}\right), \quad \widetilde{u}^{ \pm}=\left(u_{N}^{ \pm}, u_{2}^{ \pm} \partial_{1} \Phi^{ \pm}\right), \quad u_{N}^{ \pm}=\Gamma^{ \pm} v_{N}^{ \pm} .
$$

Рассматривая (47) на границе $x_{1}=0$ и учитывая последнее граничное условие в (37), получаем

$$
\frac{\Gamma^{ \pm}}{\gamma\left(\bar{p}+p^{ \pm}\right)} \partial_{0}^{ \pm} p^{ \pm}+v^{ \pm} \cdot \partial_{t} u^{ \pm} \mp v_{N}^{ \pm}\left(v^{ \pm} \cdot \partial_{1} u^{ \pm}\right) \pm \operatorname{div} \widetilde{u}^{ \pm}=0 \quad \text { при } x_{1}=0,
$$

где $\partial_{0}^{ \pm}:=\partial_{t}+v_{2}^{ \pm} \partial_{2}$. Складывая последние уравнения и используя непрерывность давления и скорости (см. (37)), приходим к равенству

$$
\left[\partial_{1} u_{N}\right]-v_{N}^{+}\left(v^{ \pm} \cdot\left[\partial_{1} u\right]\right)=0 \text { при } x_{1}=0
$$

которое с учетом

$$
\partial_{1} u^{ \pm}=\Gamma^{ \pm}\left(\partial_{1} v^{ \pm}+\left(u^{ \pm} \cdot \partial_{1} v^{ \pm}\right) u^{ \pm}\right)
$$

сводится к $\Gamma_{\mid x_{1}=0}^{+}\left[\partial_{1} v_{N}\right]=0$. Таким образом, получаем

$$
\left[\partial_{1} v_{N}\right]=0
$$

Вместо третьего, четвертого и пятого уравнений систем (34) можно использовать эквивалентные им уравнения Максвелла (5) в областях $\Omega^{ \pm}(t)$ с учетом замены переменных (22), (23). Точнее сказать, если подсистемы систем (34), состоящие из их третьих, четвертых и пятых уравнений, домножить слева на невырожденные матрицы $\mathcal{M}^{-1}\left(U^{ \pm}\right)$(матрица $\mathcal{M}$ была выписана после (8)), а затем от неизвестных $u^{ \pm}$перейти к $v^{ \pm}$, то как раз и получим упомянутые эквивалентные уравнения. Переходя в них к скачку, имеем

$$
\left[\partial_{0} H-H_{N} \partial_{1} v-H_{2} \partial_{2} v+\left(\partial_{1} v_{N}\right) H\right]=0
$$

т. е. с учетом (37) и (40)

$$
H_{N}^{+}\left[\partial_{1} v\right]=\left[\partial_{1} v_{N}\right] H^{+} \text {при } x_{1}=0 .
$$

В силу равенства (48) и условия (30) получаем (45). Наконец, рассматривая на границе сумму дивергенций (39), приходим к равенству (46). 


\section{§3. Основной результат и его обсуждение}

Мы теперь готовы сформулировать основной результат настоящей статьи, а именно локальную по времени теорему существования и единственности решения задачи (34)-(36). Очевидно, что из этой теоремы следует соответствующая теорема для исходной задачи со свободной границей (17)-(19).

Теорема 3.1. Пусть $m \in \mathbb{N}$ и $m \geq 6$. Предположим, что начальные данные (36) со свойством

$$
\left(\left(U_{0}^{+}, U_{0}^{-}\right), \varphi_{0}\right) \in H^{m+17 / 2}(\Omega) \times H^{m+17 / 2}(\partial \Omega)
$$

удовлетворяют условию гиперболичности (38), требованию (29) и дивергентным ограничениям (39) для всех $x \in \Omega$. Пусть начальные данные также удовлетворяют требованию (30), условию Рэлея - Тейлора (31) и ограничению (40) для всех $x \in \partial \Omega$. Предположим также, что начальные данные удовлетворяют условиям согласования порядка $m+8$, аналогичным условиям в [18]. Тогда существует такое достаточно малое время $T>0$, что задача (34)-(36) имеет единственное решение

$$
\left(\left(U^{+}, U^{-}\right), \varphi\right) \in H^{m}([0, T] \times \Omega) \times H^{m}([0, T] \times \partial \Omega) .
$$

В теореме используются стандартные обозначения для соболевских пространств $H^{m}:=W_{2}^{m}$, которые являются гильбертовыми пространствами.

Граничные условия (37) совпадают с граничными условиями для контактного разрыва в нерелятивистской МГД [17; 18]. Как отмечалось в [17], для таких граничных условий символ свободной границы не эллиптический. Это означает, что граничные условия не разрешимы для $\nabla_{t, x} \varphi=$ $\left(\partial_{t} \varphi, \partial_{2} \varphi\right)$. Следствием этого факта является то, что соответствующая линеаризованная задача с постоянными коэффициентами не удовлетворяет равномерному условию Лопатинского, т. е. условие Лопатинского для нее выполнено только в слабом смысле (см., например, [2]). Это, в свою очередь, влечет так называемую потерю производных в априорных оценках решения линеаризованной задачи (как с постоянными, так и с переменными коэффициентами) относительно начальных данных и правых частей этой задачи. В таких случаях не всегда, но, как правило, теорема существования решений исходной нелинейной задачи доказывается с помощью итераций Нэша - Мозера, как это и сделано в [18]. При этом начальные данные этой задачи предполагаются более гладкими, чем сами решения (см. теорему 3.1).

Интересно отметить, что, как и в нерелятивистской МГД [17; 18], магнитное поле не входит в условие Рэлея - Тейлора (31) в отличие, скажем, 
от задачи со свободной границей плазма-вакуум, для которой такое условие выписывается в [27] для полного давления $q$ (в нерелятивистской МГД $\left.q=p+|H|^{2} / 2\right)$; т. е. для контактных разрывов условие Рэлея - Тейлора возникает в его классическом (чисто «гидродинамическом») виде.

Естественно предполагать, что выполнение условия Рэлея - Тейлора в начальный момент времени гарантирует локальное существование контактного разрыва и в общем трехмерном случае. Однако вопрос о доказательстве соответствующей теоремы существования и единственности пока остается открытым и для технически более простого нерелятивистского случая (см. обсуждение в [17]).

Естественно также возникает вопрос о том, является ли условие Рэлея - Тейлора (помимо других предположений на начальные данные в теореме 3.1 и аналогичной теореме в [18]) не только достаточным, но и необходимым для корректности задачи со свободной границей. Как отмечалось в [28], неустойчивость типа Рэлея - Тейлора, в частности, означает некорректность по Адамару соответствующей линеаризованной задачи с «замороженными» коэффициентами (см. по этому поводу также интересный обзор [14]). Формально нет общего результата, что такая некорректность влечет некорректность также исходной нелинейной задачи со свободной границей, однако есть, по крайней мере, два примера задач, для которых имеется строгое доказательство того, что неустойчивость Рэлея - Тейлора влечет некорректность нелинейной задачи. Это задачи со свободными границами в идеальной несжимаемой [10] и сжимаемой жидкости [13].

Для контактного разрыва вопрос о построении примера некорректности типа примера Адамара для линеаризованной задачи с «замороженными» коэффициентами при нарушении условия Рэлея - Тейлора остается пока открытым даже для нерелятивистской МГД ввиду больших технических трудностей. Технические трудности вызваны прежде всего тем, что на контактном разрыве магнитное поле нигде не параллельно его поверхности. Так, например, для задачи со свободной границей плазма-вакуум, для которой магнитное поле на границе как раз ей всегда параллельно, в [28] удалось построить пример некорректности для соответствующей линеаризованной задачи с «замороженными» коэффициентами при одновременном нарушении условия Рэлея - Тейлора для полного давления $q$ и условия неколлинеарности магнитных полей на границе (со стороны плазмы и со стороны вакуума; см. [21;27; 28]).

Что касается сильных разрывов в РМГД, то, как и в нерелятивистской МГД $[2 ; 5]$, помимо рассматриваемых нами контактных разрывов имеются ударные волны $(\mathfrak{j} \neq 0,[\rho] \neq 0$; см. $(10)-(15))$, тангенциальные разрывы $\left(\mathfrak{j}=0, H_{n}=0\right)$ и вращательные разрывы $(\mathfrak{j} \neq 0,[\rho]=0)$. Для ударных волн в РМГД, в отличие от нерелятивистского случая (см. [2; 23]), 
пока известен только результат работы [22], где найдены области равномерной устойчивости, нейтральной устойчивости и неустойчивости параллельных быстрых ударных волн. Для тангенциальных разрывов в РМГД в [11] с помощью использования идей работ [24; 25] найдено достаточное условие «устойчивости», выполнение которого в каждой точке разрыва в начальный момент времени (помимо условий согласования и т. д.) дает локальную по времени теорему существования и единственности. Возвращаясь к контактным разрывам в РМГД, отметим, что теорема 3.1 полностью аналогична теореме существования и единственности в [18] для нерелятивистского случая.

\section{$\S 4$. Базовая априорная оценка для линеаризованной задачи}

Чтобы доказать теорему 3.1 для нелинейной задачи (34)-(36) с помощью итераций Нэша - Мозера (см., например, $[18 ; 21 ; 25 ; 26])$, необходимо провести детальный анализ соответствующей линеаризованной задачи с переменными коэффициентами. Линеаризованная задача для контактного разрыва в РМГД не имеет принципиальных отличий от соответствующей задачи в нерелятивистском случае. Однако необходимо будет обсудить специфику релятивистского случая для некоторых принципиальных моментов доказательства корректности линеаризованной задачи. Поэтому для удобства читателя мы выпишем здесь эту линеаризованную задачу.

Пусть

$$
\begin{array}{ll}
\Omega_{T}:=(-\infty, T] \times \Omega, & \partial \Omega_{T}:=(-\infty, T] \times \partial \Omega, \\
\Omega_{T}^{+}:=[0, T] \times \Omega, & \partial \Omega_{T}^{+}:=[0, T] \times \partial \Omega .
\end{array}
$$

Рассмотрим основное состояние

$$
\left(\widehat{U}^{+}(t, x), \widehat{U}^{-}(t, x), \widehat{\varphi}\left(t, x^{\prime}\right)\right)
$$

относительно которого будет проведена линеаризация. Пусть

$$
\widehat{U}^{ \pm}=\left(\widehat{p}^{ \pm}, \widehat{u}^{ \pm}, \widehat{H}^{ \pm}, \widehat{S}^{ \pm}\right)
$$

и $\widehat{\varphi}$ - достаточно гладкие функции и

$$
\left\|\widehat{U}^{+}\right\|_{W_{\infty}^{2}\left(\Omega_{T}\right)}+\left\|\widehat{U}^{-}\right\|_{W_{\infty}^{2}\left(\Omega_{T}\right)}+\|\widehat{\varphi}\|_{W_{\infty}^{2}\left(\partial \Omega_{T}\right)} \leq K,
$$

где $K>0$ - некоторая постоянная. Мы будем использовать обозначения

$$
\widehat{\Phi}^{ \pm}(t, x)= \pm x_{1}+\widehat{\Psi}^{ \pm}(t, x), \quad \widehat{\Psi}^{ \pm}(t, x)=\chi\left( \pm x_{1}\right) \widehat{\varphi}\left(t, x^{\prime}\right),
$$


т. е. все величины с крышечками определяются аналогично соответствующим величинам для $\left(U^{+}, U^{-}, \varphi\right)$, например,

$$
\begin{gathered}
\widehat{v}_{N}^{ \pm}=\widehat{v}_{1}^{ \pm}-\widehat{v}_{2}^{ \pm} \partial_{2} \widehat{\Psi}^{ \pm}, \quad \widehat{u}^{ \pm}=\widehat{\Gamma}^{ \pm} \widehat{v}^{ \pm}, \\
\widehat{\Gamma}^{ \pm}=\sqrt{1+\left|\widehat{u}^{ \pm}\right|^{2}}, \quad \widehat{H}_{\tau}^{ \pm}=\widehat{H}_{1}^{ \pm} \partial_{2} \widehat{\Psi}^{ \pm}+\widehat{H}_{2}^{ \pm} .
\end{gathered}
$$

Более того, не нарушая общности, будем предполагать, что $\|\widehat{\varphi}\|_{L_{\infty}\left(\partial \Omega_{T}\right)}<1$ (см. (24)). Из этого следуют $\partial_{1} \widehat{\Phi}^{+} \geq 1 / 2$ и $\partial_{1} \widehat{\Phi}^{-} \leq-1 / 2$.

Будем предполагать, что основное состояние удовлетворяет «ослабленному» условию гиперболичности (38), а именно неравенству

$$
\widehat{p}^{ \pm} \geq-\bar{p} / 2 \quad \text { в } \quad \Omega_{T}^{+}
$$

«ослабленному» требованию (29) недостижимости скорости света, а именно неравенству

$$
1-\left|\widehat{v}^{ \pm}\right| \geq \nu / 2>0 \quad \text { в } \Omega_{T}^{+},
$$

граничным условиям (37) вместе с ограничением (15), т. е. условиям на границе

$$
[\widehat{p}]=0, \quad[\widehat{v}]=0, \quad[\widehat{H}]=0, \quad \partial_{t} \widehat{\varphi}=\left.\widehat{v}_{N}^{+}\right|_{x_{1}=0} \quad \text { на } \partial \Omega_{T},
$$

«ослабленному» требованию (30), а именно условию

$$
\left|\widehat{H}_{N}^{ \pm}\right|_{x_{1}=0} \mid \geq \kappa / 2>0 \text { на } \partial \Omega_{T}^{+},
$$

а также условиям на скачках (45) и (46):

$$
\left[\partial_{1} \widehat{v}\right]=0, \quad\left[\partial_{1} \widehat{H}_{N}\right]=0 \text { на } \partial \Omega_{T} .
$$

Линеаризация уравнений (34) и (35) относительно состояния (49) имеет следующий вид:

$$
\begin{aligned}
\mathbb{L}^{\prime}\left(\widehat{U}^{ \pm}, \widehat{\Psi}^{ \pm}\right)\left(\delta U^{ \pm}, \delta \Psi^{ \pm}\right) & :=\left.\frac{d}{d \varepsilon} \mathbb{L}\left(U_{\varepsilon}^{ \pm}, \Psi_{\varepsilon}^{ \pm}\right)\right|_{\varepsilon=0}=f^{ \pm} \text {в } \Omega_{T}, \\
\mathbb{B}^{\prime}\left(\widehat{U}^{+}, \widehat{U}^{-}, \widehat{\varphi}\right)\left(\delta U^{+}, \delta U^{-}, \delta \varphi\right) & :=\left.\frac{d}{d \varepsilon} \mathbb{B}\left(U_{\varepsilon}^{+}, U_{\varepsilon}^{-}, \varphi_{\varepsilon}\right)\right|_{\varepsilon=0}=g \text { на } \partial \Omega_{T},
\end{aligned}
$$

где $U_{\varepsilon}^{ \pm}=\widehat{U}^{ \pm}+\varepsilon \delta U^{ \pm}, \quad \varphi_{\varepsilon}=\widehat{\varphi}+\varepsilon \delta \varphi$ и

$$
\begin{gathered}
\Psi_{\varepsilon}^{ \pm}(t, x):=\chi\left( \pm x_{1}\right) \varphi_{\varepsilon}\left(t, x^{\prime}\right), \quad \Phi_{\varepsilon}^{ \pm}(t, x):= \pm x_{1}+\Psi_{\varepsilon}^{ \pm}(t, x), \\
\delta \Psi^{ \pm}(t, x):=\chi\left( \pm x_{1}\right) \delta \varphi(t, x) .
\end{gathered}
$$


Здесь мы вводим в рассмотрение правые части

$$
f^{ \pm}(t, x)=\left(f_{1}^{ \pm}(t, x), \ldots, f_{6}^{ \pm}(t, x)\right) \quad \text { и } g\left(t, x^{\prime}\right)=\left(g_{1}\left(t, x^{\prime}\right), \ldots, g_{5}\left(t, x^{\prime}\right)\right),
$$

чтобы сделать системы и граничные условие неоднородными.

Нетрудно получить конкретный вид линеаризованных уравнений (ниже мы опускаем $\delta$ при обозначении возмущений):

$$
\begin{aligned}
& \mathbb{L}^{\prime}\left(\widehat{U}^{ \pm}, \widehat{\Psi}^{ \pm}\right)\left(U^{ \pm}, \Psi^{ \pm}\right) \\
& =L\left(\widehat{U}^{ \pm}, \widehat{\Psi}^{ \pm}\right) U^{ \pm}+\mathcal{C}\left(\widehat{U}^{ \pm}, \widehat{\Psi}^{ \pm}\right) U^{ \pm}-\left\{L\left(\widehat{U}^{ \pm}, \widehat{\Psi}^{ \pm}\right) \Psi^{ \pm}\right\} \frac{\partial_{1} \widehat{U} \pm}{\partial_{1} \widehat{\Phi}^{ \pm}} \\
& \mathbb{B}^{\prime}\left(\widehat{U}^{+}, \widehat{U}^{-}, \widehat{\varphi}\right)\left(U^{+}, U^{-}, \varphi\right)=\left(\begin{array}{c}
p^{+}-p^{-} \\
v^{+}-v^{-} \\
H_{\tau}^{+}-H_{\tau}^{-} \\
\partial_{t} \varphi+\hat{v}_{2}^{+} \partial_{2} \varphi-v_{N}^{+}
\end{array}\right),
\end{aligned}
$$

где

$$
v_{N}^{ \pm}=v_{1}^{ \pm}-v_{2}^{ \pm} \partial_{2} \widehat{\Psi}^{ \pm}, \quad H_{\tau}^{ \pm}=H_{1}^{ \pm} \partial_{2} \widehat{\Psi}^{ \pm}+H_{2}^{ \pm}
$$

и матрица $\mathcal{C}\left(\widehat{U}^{ \pm}, \widehat{\Psi}^{ \pm}\right)$определяется так:

$$
\begin{aligned}
\mathcal{C}\left(\widehat{U}^{ \pm}, \widehat{\Psi}^{ \pm}\right) Y=( & \left.Y, \nabla_{y} A_{0}\left(\widehat{U}^{ \pm}+\bar{U}^{ \pm}\right)\right) \partial_{t} \widehat{U}^{ \pm} \\
& +\left(Y, \nabla_{y} \widetilde{A}_{1}\left(\widehat{U}^{ \pm}+\bar{U}^{ \pm}, \widehat{\Psi}^{ \pm}\right)\right) \partial_{1} \widehat{U}^{ \pm} \\
& +\left(Y, \nabla_{y} A_{2}\left(\widehat{U}^{ \pm}+\bar{U}^{ \pm}\right)\right) \partial_{2} \widehat{U}^{ \pm} \\
\left(Y, \nabla_{y} A\left(\widehat{U}^{ \pm}+\bar{U}^{ \pm}\right)\right):= & \sum_{i=1}^{6} y_{i}\left(\left.\frac{\partial A(Y)}{\partial y_{i}}\right|_{Y=\widehat{U}^{ \pm}+\bar{U}^{ \pm}}\right), \quad Y=\left(y_{1}, \ldots, y_{6}\right) .
\end{aligned}
$$

Заметим также, что $v^{ \pm}$выражаются через $u^{ \pm}$следующим образом:

$$
v^{ \pm}=\frac{1}{\widehat{\Gamma}^{ \pm}}\left(u^{ \pm}-\left(\widehat{v}^{ \pm} \cdot u^{ \pm}\right) \widehat{v}^{ \pm}\right)
$$

Напомним, что мы опустили $\delta$ при обозначении возмущений, т. е. здесь $v^{ \pm}:=\delta v^{ \pm}$и $u^{ \pm}:=\delta u^{ \pm}$.

Дифференциальный оператор $\mathbb{L}^{\prime}\left(\widehat{U}^{ \pm}, \widehat{\Psi}^{ \pm}\right)$имеет первый порядок по $\Psi^{ \pm}$. Чтобы преодолеть связанную с этим фактом потенциальную трудность, мы переходим к так называемым «хорошим неизвестным» [6]:

$$
\dot{U}^{ \pm}:=U^{ \pm}-\frac{\Psi^{ \pm}}{\partial_{1} \widehat{\Phi}^{ \pm}} \partial_{1} \widehat{U}^{ \pm}
$$


Опуская выкладки, получаем линейные системы для $\dot{U}^{+}$и $\dot{U}^{-}$:

$$
L\left(\widehat{U}^{ \pm}, \widehat{\Psi}^{ \pm}\right) \dot{U}^{ \pm}+\mathcal{C}\left(\widehat{U}^{ \pm}, \widehat{\Psi}^{ \pm}\right) \dot{U}^{ \pm}-\frac{\Psi^{ \pm}}{\partial_{1} \widehat{\Phi}^{ \pm}} \partial_{1}\left\{\mathbb{L}\left(\widehat{U}^{ \pm}, \widehat{\Psi}^{ \pm}\right)\right\}=f^{ \pm}
$$

Опуская, как и в $[6 ; 17 ; 18 ; 21 ; 25 ; 26]$, младшие члены для $\Psi^{+}$и $\Psi^{-}$в системах (58), получаем «эффективные» линейные операторы

$$
\begin{aligned}
\mathbb{L}_{e}^{\prime}\left(\widehat{U}^{ \pm}, \widehat{\Psi}^{ \pm}\right) \dot{U}^{ \pm}:= & L\left(\widehat{U}^{ \pm}, \widehat{\Psi}^{ \pm}\right) \dot{U}^{ \pm}+\mathcal{C}\left(\widehat{U}^{ \pm}, \widehat{\Psi}^{ \pm}\right) \dot{U}^{ \pm} \\
= & A_{0}\left(\widehat{U}^{ \pm}+\bar{U}^{ \pm}\right) \partial_{t} \dot{U}^{ \pm}+\widetilde{A}_{1}\left(\widehat{U}^{ \pm}+\bar{U}^{ \pm}, \widehat{\Psi}^{ \pm}\right) \partial_{1} \dot{U}^{ \pm} \\
& +A_{2}\left(\widehat{U}^{ \pm}+\bar{U}^{ \pm}\right) \partial_{2} \dot{U}^{ \pm}+\mathcal{C}\left(\widehat{U}^{ \pm}, \widehat{\Psi}^{ \pm}\right) \dot{U}^{ \pm}
\end{aligned}
$$

В последующем нелинейном анализе опущенные младшие члены в (58) должны рассматриваться как дополнительные ошибки итераций Нэша Мозера.

Что касается граничного дифференциального оператора $\mathbb{B}^{\prime}$, то в терминах неизвестных (57) он принимает вид

$$
\begin{aligned}
\mathbb{B}_{e}^{\prime}(\widehat{U}, \widehat{\varphi})(\dot{U}, \varphi):= & \mathbb{B}^{\prime}(\widehat{U}, \widehat{\varphi})\left(U^{+}, U^{-}, \varphi\right) \\
= & \left(\begin{array}{c}
\dot{p}^{+}-\dot{p}^{-}+\varphi\left[\partial_{1} \widehat{p}\right] \\
\dot{v}_{1}^{+}-\dot{v}_{1}^{-} \\
\dot{v}_{2}^{+}-\dot{v}_{2}^{-} \\
\dot{H}_{\tau}^{+}-\dot{H}_{\tau}^{-}+\varphi\left[\partial_{1} \widehat{H}_{\tau}\right] \\
\partial_{t} \varphi+\widehat{v}_{2}^{+} \partial_{2} \varphi-\dot{v}_{N}^{+}-\varphi \partial_{1} \widehat{v}_{N}^{+}
\end{array}\right),
\end{aligned}
$$

где

$\widehat{U}=\left(\widehat{U}^{+}, \widehat{U}^{-}\right), \quad \dot{U}=\left(\dot{U}^{+}, \dot{U}^{-}\right), \quad \dot{v}_{N}^{ \pm}=\dot{v}_{1}^{ \pm}-\dot{v}_{2}^{ \pm} \partial_{2} \widehat{\Psi}^{ \pm}, \quad \dot{H}_{\tau}^{ \pm}=\dot{H}_{1}^{ \pm} \partial_{2} \widehat{\Psi}^{ \pm}+\dot{H}_{2}^{ \pm}$.

Здесь было использовано важное условие $\left[\partial_{1} \widehat{v}\right]=0$. Заметим также, что из (56) и (57) следует

$$
\dot{v}^{ \pm}=\frac{1}{\widehat{\Gamma}^{ \pm}}\left(\dot{u}^{ \pm}-\left(\widehat{v}^{ \pm} \cdot \dot{u}^{ \pm}\right) \widehat{v}^{ \pm}\right)
$$

Вводя обозначение

$$
\mathbb{L}_{e}^{\prime}(\widehat{U}, \widehat{\Psi}) \dot{U}:=\left(\begin{array}{l}
\mathbb{L}_{e}^{\prime}\left(\widehat{U}^{+}, \widehat{\Psi}^{+}\right) \dot{U}^{+} \\
\mathbb{L}_{e}^{\prime}\left(\widehat{U}^{-}, \widehat{\Psi}^{-}\right) \dot{U}^{-}
\end{array}\right)
$$

с $\widehat{\Psi}=\left(\widehat{\Psi}^{+}, \widehat{\Psi}^{-}\right)$, мы выписываем линейную задачу для $(\dot{U}, \varphi)$ :

$$
\begin{aligned}
\mathbb{L}_{e}^{\prime}(\widehat{U}, \widehat{\Psi}) \dot{U} & =f \quad \text { в } \quad \Omega_{T}, \\
\mathbb{B}_{e}^{\prime}(\widehat{U}, \widehat{\varphi})(\dot{U}, \varphi) & =g \quad \text { на } \partial \Omega_{T}, \\
(\dot{U}, \varphi) & =0 \quad \text { при } t<0,
\end{aligned}
$$


где $f=\left(f^{+}, f^{-}\right)$. Здесь предполагается, что $f$ и $g$ равны нулю в прошлом (при $t<0)$, а начальные данные берутся однородными, так как рассмотрение случая неоднородных начальных данных может быть отложено на нелинейный анализ (построение так называемого аппроксимационного решения; см., например, [26]).

Теорема 4.1. Пусть для основного состояния (49) справедливы предположения (50)-(55) и оно удовлетворяет условию Рэлея - Тейлора

$$
\left[\partial_{1} \widehat{p}\right] \geq \epsilon / 2>0 \quad \text { на } \partial \Omega_{T}^{+}
$$

где

$$
\left[\partial_{1} \widehat{p}\right]=\partial_{1} \widehat{p}_{\mid x_{1}=0}^{+}+\partial_{1} \widehat{p}_{\mid x_{1}=0}^{-}
$$

(cм. (32)). Тогда для всех $f \in H^{2}\left(\Omega_{T}\right)$ и $g \in H^{2}\left(\partial \Omega_{T}\right)$, равных нулю в прошлом, задача (60)-(62) имеет единственное решение

$$
(\dot{U}, \varphi) \in H^{1}\left(\Omega_{T}\right) \times H^{1}\left(\partial \Omega_{T}\right) .
$$

Более того, это решение удовлетворяет априорной оценке

$$
\|\dot{U}\|_{H^{1}\left(\Omega_{T}\right)}+\|\varphi\|_{H^{1}\left(\partial \Omega_{T}\right)} \leq C\left\{\|f\|_{H^{2}\left(\Omega_{T}\right)}+\|g\|_{H^{2}\left(\partial \Omega_{T}\right)}\right\},
$$

где $C=C(K, \bar{p}, \kappa, \epsilon, T)>0-$ постоянная, не зависящая от данных $f$ и $g$.

Отсылая читателя к подробному доказательству соответствующей теоремы в [17] для нерелятивистской МГД, прокомментируем коротко доказательство теоремы 4.1, останавливаясь чуть более подробно на местах, которые требуют определенного внимания в связи со спецификой релятивистского случая. Мы будем также параллельно ссылаться на работу [18], где априорные оценки линеаризованной задачи выводились в старших нормах, но сам процесс вывода этих оценок не принципиально, но немного отличается от получения базовой априорной $H^{1}$-оценки в [17].

Заметим, что в [17] в правой части аналога априорной оценки (64) фигурируют нормы $\|f\|_{H^{1}\left(\Omega_{T}\right)}$ и $\|g\|_{H^{3 / 2}\left(\partial \Omega_{T}\right)}$. Во-первых, мы сознательно «загрубили» оценку (64), написав вместо $\|g\|_{H^{3 / 2}\left(\partial \Omega_{T}\right)}$ норму $\|g\|_{H^{2}\left(\partial \Omega_{T}\right)}$, поскольку «выигрыш половинки производной» никак не используется при доказательстве теоремы существования для нелинейной задачи. Во-вторых, что касается нормы правой части $f$, то действительно специфика нашего релятивистского случая такова, что в смысле бо́льших требований на правую часть $f$ оценка (64) чуть хуже аналогичной оценки из [17]. Однако это никак не влияет на конечный результат теоремы 3.1 для нелинейной задачи. Гладкость начальных данных и решения в теореме 3.1 та же самая, что и в аналогичной теореме из [18]. Как отмечалось в [26], меньшие 
требования на гладкость правой части $f$ по сравнению с требованиями для $g$ не имеют никакого значения при доказательстве сходимости итераций Нэша - Мозера; т. е. в итоге все равно используется априорная оценка, в правой части которой показатель соболевских пространств для норм вектор-функций $f$ и $g$ один и тот же.

Сперва с помощью стандартного рассуждения граничные условия сводятся к однородным (см. [17; 18]). Однако для нашего релятивистского случая следует слегка модифицировать стандартную процедуру. Вопервых, как и в $[17 ; 18]$, мы рассматриваем некоторую вектор-функцию $\widetilde{U}=\left(\widetilde{U}^{+}, \widetilde{U}^{-}\right)$, которая удовлетворяет граничным условия (61) при $\varphi=0$ (и равна нулю при $t<0$ ). Тогда новое неизвестное

$$
\dot{U}^{\natural}=\left(U^{+\natural}, U^{-\natural}\right)=\dot{U}-\widetilde{U},
$$

для которого верна оценка

$$
\|\widetilde{U}\|_{H^{s+1}\left(\Omega_{T}\right)} \leq C\|g\|_{H^{s+1 / 2}\left(\partial \Omega_{T}\right)}
$$

(в этом параграфе $s=0$ и $s=1$ ), удовлетворяет задаче (60)-(62) с $f=$ $\widetilde{f}=\left(\tilde{f}^{+}, \tilde{f}^{-}\right)$, где

$$
\widetilde{f}^{ \pm}=f^{ \pm}-\mathbb{L}_{e}^{\prime}\left(\widehat{U}^{ \pm}, \widehat{\Psi}^{ \pm}\right) \widetilde{U}^{ \pm} .
$$

Здесь и далее $C$ - общее обозначение для положительной постоянной, которая не зависит от правых частей задачи и, в частности, может зависеть от константы $K$ из (50).

Выбирая $\widetilde{U}$, удовлетворяющее граничным условиям (61), мы имеем требование $\left[\widetilde{H}_{\tau}\right]=g_{4}$ только на тангенциальные компоненты векторов $\widetilde{H}^{ \pm}$, в то время как для их нормальных компонент $\widetilde{H}_{N}^{ \pm}$остается определенный произвол. Потребуем, чтобы последние удовлетворяли условию $\left[\widetilde{H}_{N}\right]=g_{6}$, где $g_{6}$ является решением уравнения (ниже мы обсудим почему рассматривается именно такое уравнение)

$$
\partial_{t} g_{6}+\partial_{2}\left(\widehat{v}_{2}^{+} g_{6}\right)=\left[f_{H} \cdot \widehat{N}\right] \text { на } \partial \Omega_{T},
$$

где $\widehat{N}^{ \pm}=\left(1,-\partial_{2} \widehat{\Psi}^{ \pm}\right)$, а $f_{H}^{ \pm}=\left(f_{H}^{1 \pm}, f_{H}^{2 \pm}\right)$ - правые части в уравнениях для $\dot{H}^{ \pm}$, которые являются следствием системы (60) (ниже эти уравнения $\partial_{t} \dot{H}^{ \pm}+\cdots=f_{H}^{ \pm}$будут выписаны с точностью до младших членов). Правые части $f_{H}^{ \pm}$, определяющиеся через $f^{ \pm}$и основное состояние (49), при желании могут быть выписаны, хотя их конкретный вид не имеет значения. Теперь вместо (66) мы имеем оценку

$$
\|\widetilde{U}\|_{H^{s+1}\left(\Omega_{T}\right)} \leq C\left\|\left(g, g_{6}\right)\right\|_{H^{s+1 / 2}\left(\partial \Omega_{T}\right)}
$$

(причем $\left\|\widetilde{H}_{N}^{ \pm}\right\|_{H^{s+1}\left(\Omega_{T}\right)} \leq C\left\|g_{6}\right\|_{H^{s+1 / 2}\left(\partial \Omega_{T}\right)}$ ). 
Используя теорему о следе на границе для функций $f_{H}^{ \pm}$, из уравнения (68) выводим оценку

$$
\left\|g_{6}\right\|_{H^{3 / 2}\left(\partial \Omega_{T}\right)} \leq C\left\|\left.f\right|_{x_{1}=0}\right\|_{H^{3 / 2}\left(\partial \Omega_{T}\right)} \leq C\|f\|_{H^{2}\left(\Omega_{T}\right)} .
$$

Тогда для $\widetilde{H}_{N}^{ \pm}$имеем

$$
\left\|\widetilde{H}_{N}^{ \pm}\right\|_{H^{2}\left(\Omega_{T}\right)} \leq C\left\|g_{6}\right\|_{H^{3 / 2}\left(\partial \Omega_{T}\right)} \leq C\|f\|_{H^{2}\left(\Omega_{T}\right)} .
$$

Учитывая (69) (при $s=1$ ), окончательно получаем следующую оценку для $\widetilde{U}$ :

$$
\|\widetilde{U}\|_{H^{2}\left(\Omega_{T}\right)} \leq C\left(\|g\|_{H^{3 / 2}\left(\partial \Omega_{T}\right)}+\|f\|_{H^{2}\left(\Omega_{T}\right)}\right) .
$$

Понятно, что в силу (70) для новой правой части $\widetilde{f}($ см. (67)) справедлива оценка

$$
\|\tilde{f}\|_{H^{1}\left(\Omega_{T}\right)} \leq C\left(\|g\|_{H^{2}\left(\partial \Omega_{T}\right)}+\|f\|_{H^{2}\left(\Omega_{T}\right)}\right) .
$$

Мы «загрубили» эту оценку, написав в правой ее части норму $\|g\|_{H^{2}\left(\partial \Omega_{T}\right)}$ вместо нормы $\|g\|_{H^{3 / 2}\left(\partial \Omega_{T}\right)}$.

Что касается уравнения (68), то в [17] показано, что именно этому уравнению удовлетворяет скачок $\left[\dot{H}_{N}\right]$ (при $\left.g=0\right)$. Тогда после сдвига неизвестных (65) получаем уравнение

$$
\partial_{t}\left[\dot{H}_{N}^{\natural}\right]+\partial_{2}\left(\widehat{v}_{2}^{+}\left[\dot{H}_{N}^{\natural}\right]\right)=0 \text { на } \partial \Omega_{T},
$$

из которого ввиду однородности начальных данных следует $\left[\dot{H}_{N}^{\natural}\right]=0$.

Таким образом, опуская после сдвига неизвестных (65) верхние индексы $\downarrow$ и «волны» над $\tilde{f}$ и $\widetilde{f}^{ \pm}$, получаем задачу (60)-(62) с $g=0$, решения которой удовлетворяют дополнительному условию на границе

$$
\left[\dot{H}_{N}\right]=0 .
$$

Для этой задачи с однородными граничными условиями и дополнительным условием (72) нам нужно получить априорную оценку

$$
\|\dot{U}\|_{H^{1}\left(\Omega_{T}\right)}+\|\varphi\|_{H^{1}\left(\partial \Omega_{T}\right)} \leq C\|f\|_{H^{1}\left(\Omega_{T}\right)} .
$$

Тогда с учетом неравенств (70) и (71) из (73) будет следовать искомая априорная оценка (64).

Для вывода априорной оценки (73), как и в [17], мы вначале получим вспомогательную оценку

$$
\left\|\partial_{1} \dot{U}(t)\right\|_{L^{2}(\Omega)}^{2} \leq C\left\{\|f\|_{H^{1}\left(\Omega_{T}\right)}^{2}+\||| \dot{U}(t) \mid\|_{\tan , 1}^{2}+\int_{0}^{t}\|\dot{U}(s)\|_{H^{1}(\Omega)}^{2} \mathrm{~d} s\right\},
$$


где

$$
\begin{aligned}
& \left.\|\dot{U}(t)\|\right|_{\tan , 1} ^{2}:=\|\dot{U}(t)\|_{L^{2}(\Omega)}^{2}+\left\|\partial_{t} \dot{U}(t)\right\|_{L^{2}(\Omega)}^{2}+\left\|\partial_{2} \dot{U}(t)\right\|_{L^{2}(\Omega)}^{2}, \\
& \|\dot{U}(s)\|\left\|_{H^{1}(\Omega)}^{2}:=\right\| \dot{U}(t)\left\|_{\tan , 1}^{2}+\right\| \partial_{1} \dot{U}(t) \|_{L^{2}(\Omega)}^{2} .
\end{aligned}
$$

Здесь и далее оценками мы называем некоторые вспомогательные неравенства, которые не являются априорными оценками в обычном смысле. Для получения оценки (74) нужно воспользоваться структурой граничной матрицы $\mathfrak{A}_{1}$ (см. (41)), выписанной на основном состоянии (49), и предположением (54). В отличие от гораздо более технически простого нерелятивистского случая, нам непросто явно выписать граничную матрицу, как это сделано в $[17 ; 18]$. Но, к счастью, этого и не требуется.

Действительно, граничные матрицы $\pm\left.\widetilde{A}_{1}\left(\widehat{U}^{ \pm}, \widehat{\Psi}^{ \pm}\right)\right|_{x_{1}=0}$ имеют по два нулевых собственных числа (см. (44)). Вначале нужно понять, каким неизвестным (будем называть их характеристическими) соответствуют эти нулевые собственные числа. Во-первых, такими характеристическими неизвестными являются $\dot{S}^{ \pm}$(так же, как и в $\left.[17 ; 18]\right)$. В самом деле, так как последнее уравнение в (8) есть $\mathrm{d} S / \mathrm{d} t=0$, его линеаризация с учетом предварительной замены переменных (22), (23) и последующей замены неизвестных (57) дает шестое и двенадцатое уравнения системы (60):

$$
\partial_{t} \dot{S}^{ \pm}+\frac{1}{\partial_{1} \widehat{\Phi}^{ \pm}}\left(\widehat{w}^{ \pm} \cdot \nabla \dot{S}^{ \pm}\right)+\text {l.o.t. }=f_{6}^{ \pm}
$$

где $\widehat{w}^{ \pm}$- это векторы $w^{ \pm}$, фигурирующие в (47), но выписанные на основном состоянии (49). Здесь и далее l.o.t. - общее обозначение для младших членов, конкретный вид которых не имеет значения. В силу последнего предположения в (53) имеем $\left.\widehat{w}_{1}^{ \pm}\right|_{x_{1}=0}=0$; т. е. $\dot{S}^{ \pm}$действительно являются характеристическими неизвестными.

Что касается еще двух характеристических неизвестных, то, как и в нерелятивистской МГД [17; 18], ими являются $\dot{H}_{N}^{ \pm}=\dot{H}_{1}^{ \pm}-\dot{H}_{2}^{ \pm} \partial_{2} \widehat{\Psi}^{ \pm}$. Действительно, линеаризация системы (5) с учетом предварительной замены переменных (22), (23) и последующей замены неизвестных (57) дает уравнения

$$
\partial_{t} \dot{H}^{ \pm}+\frac{1}{\partial_{1} \widehat{\Phi}^{ \pm}}\left\{\left(\widehat{w}^{ \pm} \cdot \nabla\right) \dot{H}^{ \pm}-\left(\widehat{\mathfrak{h}}^{ \pm} \cdot \nabla\right) \dot{v}^{ \pm}+\widehat{H}^{ \pm} \operatorname{div} \widetilde{\dot{v}}^{ \pm}\right\}+\text {l.o.t. }=f_{H}^{ \pm},
$$

которые являются следствием системы (60), где

$$
\tilde{\dot{v}}^{ \pm}=\left(\dot{v}_{N}^{ \pm}, \dot{v}_{2}^{ \pm} \partial_{1} \widehat{\Phi}^{ \pm}\right)
$$


(см. также (59)). В свою очередь, из (76) следует

$$
\partial_{t} \dot{H}_{N}^{ \pm}+\frac{1}{\partial_{1} \widehat{\Phi}^{ \pm}}\left(\widehat{w}^{ \pm} \cdot \nabla \dot{H}_{N}^{ \pm}\right)-\widehat{H}_{2}^{ \pm} \partial_{2} \dot{v}_{N}^{ \pm}+\widehat{H}_{N}^{ \pm} \partial_{2} \dot{v}_{2}^{ \pm}+\text {l.o.t. }=\left(f_{H}^{ \pm} \cdot \widehat{N}^{ \pm}\right) .
$$

Так как $\left.\widehat{w}_{1}^{ \pm}\right|_{x_{1}=0}=0$, мы не можем разрешить последние уравнения для $\partial_{1} \dot{H}_{N}^{ \pm}$в каждой точке $\Omega_{T}$, т. е. $\dot{H}_{N}^{ \pm}$действительно являются характеристическими неизвестными.

Таким образом, неизвестные $\dot{p}^{ \pm}, \dot{u}^{ \pm}$и $\dot{H}_{2}^{ \pm}$(или $\dot{H}_{\tau}^{ \pm}$) не являются характеристическими. Из условия (54) следует строгая положительность скоростей $c_{s_{ \pm}}^{ \pm}$и $c_{f_{ \pm}}^{ \pm}$, вычисленных на основном состоянии (см. $\left.(44)\right)$. Тогда в силу предположения непрерывности основного состояния (49) мы можем разрешить систему (60) для производных по $x_{1}$ этих нехарактеристических неизвестных в некоторой малой окрестности границы $x_{1}=0$, а затем, следуя стандартным рассуждениям из [17; 18], мы в итоге получаем оценку во всей области $\Omega$ :

$$
\left\|\partial_{1} \dot{U}_{n}(t)\right\|_{L^{2}(\Omega)}^{2} \leq C\left\{\|f\|_{H^{1}\left(\Omega_{T}\right)}^{2}+\||\dot{U}(t)|\|_{\tan , 1}^{2}+\int_{0}^{t}\|\dot{U}(s) \mid\|_{H^{1}(\Omega)}^{2} \mathrm{~d} s\right\},
$$

где $\dot{U}_{n}=\left(\dot{U}_{n}^{+}, \dot{U}_{n}^{-}\right)$и $\dot{U}_{n}^{ \pm}=\left(\dot{p}^{ \pm}, \dot{u}^{ \pm}, \dot{H}_{2}^{ \pm}\right)$.

Далее, следуя [17; 18], для оценивания производных по $x_{1}$ характеристических неизвестных $\dot{H}_{N}^{ \pm}$и $\dot{S}^{ \pm}$используем уравнения $(75)$, а также уравнения для линеаризованных дивергенций магнитных полей

$$
\xi^{ \pm}=\operatorname{div} \dot{\mathfrak{h}}^{ \pm}=\partial_{1} \dot{H}_{N}^{ \pm}+\partial_{2}\left(\dot{H}_{2}^{ \pm} \partial_{1} \widehat{\Phi}^{ \pm}\right),
$$

которые получаются применением оператора $\operatorname{div}$ к системам для $\dot{\mathfrak{h}}^{ \pm}$, являющихся следствием систем (76):

$$
\partial_{t}\left(\frac{\xi^{ \pm}}{\partial_{1} \widehat{\Phi}^{ \pm}}\right)+\frac{1}{\partial_{1} \widehat{\Phi}^{ \pm}}\left(\widehat{w}^{ \pm} \cdot \nabla\left(\frac{\xi^{ \pm}}{\partial_{1} \widehat{\Phi}^{ \pm}}\right)\right)+\text {l.o.t }=\frac{\operatorname{div} f_{\mathfrak{h}}^{ \pm}}{\partial_{1} \widehat{\Phi}^{ \pm}}
$$

где

$$
f_{\mathfrak{h}}^{ \pm}=\left(f_{H}^{1 \pm}-f_{H}^{2 \pm} \partial_{2} \widehat{\Psi}^{ \pm}, f_{H}^{2 \pm} \partial_{1} \widehat{\Phi}^{ \pm}\right) .
$$

Как уравнения (75), так и уравнения (79) не требуют граничных условий на границе $x_{1}=0$, поскольку $\left.\widehat{w}_{1}^{ \pm}\right|_{x_{1}=0}=0$. Тогда, опуская стандартные рассуждения энергетического метода, в итоге получаем оценку

$$
\left\|\partial_{1} \dot{U}_{c}(t)\right\|_{L^{2}(\Omega)}^{2} \leq C\left\{\|f\|_{H^{1}\left(\Omega_{T}\right)}^{2}+\||\dot{U}(t)|\|_{\tan , 1}^{2}+\int_{0}^{t}\|\dot{U}(s)\| \|_{H^{1}(\Omega)}^{2} \mathrm{~d} s\right\},
$$

где $\dot{U}_{c}=\left(\dot{H}_{N}^{+}, \dot{H}_{N}^{-}, \dot{S}^{+}, \dot{S}^{-}\right)$. Оценки (78) и (80) дают (74). 
Как уже отмечалось выше, для контактного разрыва символ свободной границы не эллиптический. Для линейной задачи (60)-(62) это означает, что из граничных условий (61) мы не можем выразить «градиент фронта» $\left(\partial_{t} \varphi, \partial_{2} \varphi\right)$ через след $\dot{U}_{\mid x_{1}=0}$. Однако, как и в нерелятивистском случае [17; 18], последнее граничное условие разрешимо для «материальной» производной $\partial_{0} \varphi=\partial_{t} \varphi+\hat{v}_{2}^{+} \partial_{2} \varphi$ (так как $\left.\widehat{w}_{1}^{+}\right|_{x_{1}=0}=0$, то на границе производная $\partial_{0}$ совпадает с материальной производной $\partial_{t}+\left(\widehat{w}^{+} \cdot \nabla\right)$ в системе координат, движущейся вместе с разрывом). Тогда, проводя те же рассуждения, что и в $[17 ; 18]$, можно получить вспомогательную оценку

$$
\begin{aligned}
& \|\dot{U}(t)\|_{L^{2}(\Omega)}^{2}+\left\|\partial_{0} \dot{U}(t)\right\|_{L^{2}(\Omega)}^{2}+\|\varphi(t)\|_{L^{2}(\Omega)}^{2} \\
& \quad \leq \varepsilon C\|U(t)\|_{H^{1}(\Omega)}^{2}+\frac{C}{\varepsilon}\left\{\|f\|_{H^{1}\left(\Omega_{T}\right)}^{2}+\int_{0}^{t} I(s) \mathrm{d} s\right\},
\end{aligned}
$$

где $\varepsilon>0-$ произвольная постоянная (здесь $C$ не зависит от $\varepsilon$ ) и

$$
I(t)=\|\| U(t)\|\|_{H^{1}(\Omega)}^{2}+\|\varphi(t)\|_{L^{2}(\Omega)}^{2}+\left\|\partial_{t} \varphi(t)\right\|_{L^{2}(\Omega)}^{2}+\left\|\partial_{2} \varphi(t)\right\|_{L^{2}(\Omega)}^{2} .
$$

Понятно, что, имея оценки для $\partial_{0} \dot{U}$ и $\partial_{2} \dot{U}$, мы можем получить соответствующую оценку для $\partial_{t} \dot{U}$; т. е. нам не хватает оценки для $\partial_{2} \dot{U}$, а также оценок для $\partial_{t} \varphi$ и $\partial_{2} \varphi$. Комбинация этих оценок с оценками $(74)$ и (81) (при выборе достаточно малого $\varepsilon$ ) дала бы энергетическое неравенство

$$
I(t) \leq\left\{\|f\|_{H^{1}\left(\Omega_{T}\right)}^{2}+\int_{0}^{t} I(s) \mathrm{d} s\right\} .
$$

Используя лемму Гронуолла, из этого неравенства выводим априорную оценку (73). Возвращаясь затем к неоднородным граничным условиям, мы получаем априорную оценку (64).

Вывод вспомогательных оценок (74) и (81) имеет чисто технический характер и не связан с трудностями, преодоление которых требовало бы использование определенных новых идей для задачи для контактного разрыва. При выводе (74) и (81) также не используется принципиальное предположение о выполнении условия Рэлея - Тейлора (63). Получение же оценки для $\partial_{2} \dot{U}$ (попутно также получается оценка для $\partial_{2} \varphi$ ), напротив, имеет ключевое значение для вывода энергетического неравенства (82).

Вывод оценки для $\partial_{2} \dot{U}$ не имеет принципиальных отличий от вывода соответствующей оценки для нерелятивистского случая [17; 18]. Для того чтобы понять возможность адаптации рассуждений из $[17 ; 18]$ к релятивистской задаче, необходимо только уделить внимание квадратичной форме с граничной матрицей $\mathfrak{A}_{1}$ (см. (41)), выписанной на основном состоянии (49). Пусть

$$
\widehat{\mathfrak{A}}_{1}:=\left.\mathfrak{A}_{1}\right|_{U^{ \pm}=\widehat{U}^{ \pm}, \varphi=\widehat{\varphi}} .
$$


Тогда

$$
\left.\left(\widehat{\mathfrak{A}}_{1} \dot{U} \cdot \dot{U}\right)\right|_{x_{1}=0}=\left[\left(\left\{A_{1}(\widehat{U}+\bar{U})-A_{0}(\widehat{U}+\bar{U}) \partial_{t} \varphi-A_{2}(\widehat{U}+\bar{U}) \partial_{2} \varphi\right\} \dot{U} \cdot \dot{U}\right)\right]
$$

где скачок имеет вид

$$
[(\cdot)(\widehat{U}, \bar{U}, \dot{U})]=\left.(\cdot)\left(\widehat{U}^{+}, \bar{U}^{+}, \dot{U}^{+}\right)\right|_{x_{1}=0}-\left.(\cdot)\left(\widehat{U}^{-}, \bar{U}^{-}, \dot{U}^{-}\right)\right|_{x_{1}=0} .
$$

Для вычисления квадратичной формы (83) необходимо понять структуру матрицы

$$
A_{1}(U)-A_{0}(U) \partial_{t} \varphi-A_{2}(U) \partial_{2} \varphi \text { при } \partial_{t} \varphi=v_{N}:=v_{1}-v_{2} \partial_{2} \varphi .
$$

Для этого нужно использовать следующее представление матриц системы (8):

$$
A_{j}=v_{j} A_{0}+G_{j}
$$

(напомним, что мы рассматриваем двумерный случай, т. е. $j=1,2$ ), где

$$
\begin{gathered}
G_{j}=\left(\begin{array}{cccc}
0 & e_{j}^{\top}-v_{j} v^{\top} & 0 & 0 \\
e_{j}-v_{j} v & \mathcal{G}_{j} & \mathcal{N}_{j}^{\top} & 0 \\
0 & \mathcal{N}_{j} & 0 & 0 \\
0 & 0 & 0 & 0
\end{array}\right), \\
\mathcal{G}_{j}=v_{j}\left\{2 \frac{B^{2}}{\Gamma} v \otimes v-\frac{(v \cdot H)}{\Gamma}(v \otimes H+H \otimes v)\right\} \\
+\frac{H_{j}}{\Gamma}\left\{\frac{1}{\Gamma^{2}}(v \otimes H+H \otimes v)-2(v \cdot H)(I-v \otimes v)\right\} \\
+\frac{(v \cdot H)}{\Gamma}\left(H \otimes e_{j}+e_{j} \otimes H\right)-\frac{B^{2}}{\Gamma}\left(v \otimes e_{j}+e_{j} \otimes v\right) .
\end{gathered}
$$

Тогда при $\partial_{t} \varphi=v_{N}$ имеем

$$
A_{1}(U)-A_{0}(U) \partial_{t} \varphi-A_{2}(U) \partial_{2} \varphi=G_{N}(U, \varphi):=G_{1}(U)-G_{2}(U) \partial_{2} \varphi .
$$

Нетрудно понять, что

$$
G_{N}=\left(\begin{array}{cccc}
0 & N^{\top}-v_{N} v^{\top} & 0 & 0 \\
N-v_{N} v & \mathcal{G}_{N} & \mathcal{N}_{N}^{\top} & 0 \\
0 & \mathcal{N}_{N} & 0 & 0 \\
0 & 0 & 0 & 0
\end{array}\right)
$$

где $N=\left(1,-\partial_{2} \varphi\right)$, 


$$
\mathcal{N}_{N}=\frac{1}{\Gamma} b \otimes N-\frac{v_{N}}{\Gamma} b \otimes v-\frac{H_{N}}{\Gamma^{2}} I, \quad H_{N}=H_{1}-H_{2} \partial_{2} \varphi
$$

И

$$
\begin{aligned}
\mathcal{G}_{N}=v_{N}\{ & \left.2 \frac{B^{2}}{\Gamma} v \otimes v-\frac{(v \cdot H)}{\Gamma}(v \otimes H+H \otimes v)\right\} \\
& +\frac{H_{N}}{\Gamma}\left\{\frac{1}{\Gamma^{2}}(v \otimes H+H \otimes v)-2(v \cdot H)(I-v \otimes v)\right\} \\
& +\frac{(v \cdot H)}{\Gamma}(H \otimes N+N \otimes H)-\frac{B^{2}}{\Gamma}(v \otimes N+N \otimes v) .
\end{aligned}
$$

С учетом условий $\partial_{t} \widehat{\varphi}=\left.\widehat{v}_{N}^{ \pm}\right|_{x_{1}=0}($ см. (53)) получаем

$$
\left.\left(\widehat{\mathfrak{A}}_{1} \dot{U} \cdot \dot{U}\right)\right|_{x_{1}=0}=\left[\left(G_{N}(\widehat{U}, \widehat{\varphi}) \dot{U} \cdot \dot{U}\right)\right]
$$

Поскольку $[\widehat{v}]=[\widehat{H}]=0$ и $[\dot{u}]=0($ см. (53), (59) и (61) при $g=0)$, имеем

$$
\begin{aligned}
\frac{1}{2}\left[\left(G_{N}(\widehat{U}, \varphi) \dot{U} \cdot \dot{U}\right)\right] & =\left[\dot{p} \dot{u}_{N}-\widehat{v}_{N}(\widehat{v} \cdot \dot{u}) \dot{p}+\left(\widehat{\mathcal{N}}_{N} \dot{u} \cdot \dot{H}\right)\right] \\
& =\left[\widehat{\Gamma}_{\dot{p}} \dot{v}_{N}+\dot{v}_{N}(\widehat{b} \cdot \dot{H})-\left(\widehat{H}_{N} / \widehat{\Gamma}^{2}\right)(\dot{u} \cdot \dot{H})\right]
\end{aligned}
$$

где

$$
\dot{u}_{N}^{ \pm}=\dot{u}_{1}^{ \pm}-\dot{u}_{2}^{ \pm} \partial_{2} \widehat{\Psi}^{ \pm} \text {и }\left.\widehat{\mathcal{N}}_{N}^{ \pm}\right|_{x_{1}=0}=\mathcal{N}_{N}\left(\widehat{U}_{\mid x_{1}=0}^{ \pm}, \widehat{\varphi}\right) .
$$

Опуская простые вычисления, в итоге с учетом граничного условия $[\dot{v}]=0$ (см. (61) при $g=0)$ и предположений (53) получаем

$$
\begin{aligned}
& -\left.\frac{1}{2}\left(\widehat{\mathfrak{A}}_{1} \dot{U} \cdot \dot{U}\right)\right|_{x_{1}=0} \\
& \quad=\left.\widehat{\Gamma}^{+}\left\{\left(\widehat{H}_{N}^{+} \dot{v}_{2}^{+}-\widehat{H}_{2}^{+} \dot{v}_{N}^{+}\right)\left(\left(1-\hat{\sigma}^{2}\right)\left[\dot{H}_{\tau}\right]+\hat{\sigma}^{2}\left[\dot{H}_{N}\right]\right)-\dot{v}_{N}^{+}[\dot{p}]\right\}\right|_{x_{1}=0}
\end{aligned}
$$

где $\widehat{\sigma}=\partial_{t} \widehat{\varphi} / \sqrt{1+\left(\partial_{2} \widehat{\varphi}\right)^{2}}$ - скорость невозмущенного разрыва (на самом деле явный вид коэффициентов перед $\left[\dot{H}_{\tau}\right]$ и $\left[\dot{H}_{N}\right]$ не имеет значения для дальнейших рассуждений) .

Принципиальным отличием квадратичной формы (84) от соответствующей формы для нерелятивистского контактного разрыва [17; 18] является присутствие слагаемого, связанного с наличием $\left[\dot{H}_{N}\right]$. Именно по этой причине мы вынуждены были модифицировать процедуру перехода к однородным граничным условиям и именно поэтому, в отличие от [17; 18], мы 
имеем «потерю одной производной» для правой части $f$ в априорной оценке (64). Благодаря этой модификации мы имеем условие на границе (72), из которого следует

$$
-\left.\frac{1}{2}\left(\widehat{\mathfrak{A}}_{1} \dot{U} \cdot \dot{U}\right)\right|_{x_{1}=0}=\left.\widehat{\Gamma}^{+}\left\{\left(1-\widehat{\sigma}^{2}\right)\left(\widehat{H}_{N}^{+} \dot{v}_{2}^{+}-\widehat{H}_{2}^{+} \dot{v}_{N}^{+}\right)\left[\dot{H}_{\tau}\right]-\dot{v}_{N}^{+}[\dot{p}]\right\}\right|_{x_{1}=0} .
$$

Дальнейшие рассуждения полностью совпадают с рассуждениями из $[17 ; 18]$. Для удобства читателя сделаем все же несколько комментариев по поводу получения оценки для $\partial_{2} \dot{U}$. Как и в [18], удобно от неизвестного $\dot{U}$ перейти к $V=\left(V^{+}, V^{-}\right)$, где

$$
V^{ \pm}=\left(\dot{p}^{ \pm}, \dot{v}_{N}^{ \pm}, \dot{v}_{2}^{ \pm}, \dot{H}_{N}^{ \pm}, \dot{H}_{\tau}^{ \pm}, \dot{S}^{ \pm}\right)
$$

(напомним, что $\left.\dot{U}^{ \pm}=\left(\dot{p}^{ \pm}, \dot{u}^{ \pm}, \dot{H}^{ \pm}, \dot{S}^{ \pm}\right)\right)$. Переход к $V$ необязателен (он не был сделан в [17]), но удобен тем, что он упрощает вид квадратичной формы с граничной матрицей для производных от неизвестных по $x_{2}$. Нетрудно выписать матрицу перехода $J=\operatorname{diag}\left(J^{+}, J^{-}\right)$, зависящую от основного состояния (49), для которой $\dot{U}^{ \pm}=J^{ \pm} V^{ \pm}$. Однако ее конкретный вид не важен для вывода оценок. После перехода к $V$ и домножения системы (60) слева на матрицу $J^{\top}$ мы вновь имеем симметрическую гиперболическую систему. Дифференцируя эту систему по $x_{2}$ и используя стандартные рассуждения энергетического метода, описание которых мы опускаем, получаем неравенство

$$
\int_{\Omega}\left(B_{0} \partial_{2} V \cdot \partial_{2} V\right) \mathrm{d} x+2 \int_{\partial \Omega_{t}} Q \mathrm{~d} x_{2} \mathrm{~d} s \leq C\left\{\|f\|_{H^{1}\left(\Omega_{T}\right)}^{2}+\int_{0}^{t} I(s) \mathrm{d} s\right\},
$$

где $B_{0}=J^{\top} \widehat{\mathfrak{A}}_{0} J, \widehat{\mathfrak{A}}_{0}=\operatorname{diag}\left(A_{0}\left(\widehat{U}^{+}+\bar{U}^{+}\right), A_{0}\left(\widehat{U}^{-}+\bar{U}^{-}\right)\right)$и в силу $(85)$

$$
Q=-\left.\frac{1}{2}\left(\widehat{\mathfrak{A}}_{1} \partial_{2} V \cdot \partial_{2} V\right)\right|_{x_{1}=0}=\left.\widehat{\Gamma}^{+}\left\{\left(1-\hat{\sigma}^{2}\right) R\left[\partial_{2} \dot{H}_{\tau}\right]-\partial_{2} \dot{v}_{N}^{+}\left[\partial_{2} \dot{p}\right]\right\}\right|_{x_{1}=0},
$$

где

$$
R=\left.\left(\widehat{H}_{N}^{+} \partial_{2} \dot{v}_{2}^{+}-\partial_{2} \widehat{H}_{2}^{+} \dot{v}_{N}^{+}\right)\right|_{x_{1}=0} .
$$

Далее, следуя [17; 18], из $(77)$ при $x_{1}=0$ находим

$$
R=-\partial_{0} \dot{H}_{N}^{+}+\left(f_{H}^{+} \cdot \widehat{N}^{+}\right)+\text {l.o.t. на } \partial \Omega_{T} .
$$

Используя первое, четвертое и пятое граничные условия из (61) (при $g=0$ ), а также подставляя в квадратичную форму $Q$ выражение для $R$ из (87), получаем

$$
Q=\left.\frac{1}{2} \partial_{t}\left\{\widehat{\Gamma}^{+}\left[\partial_{1} \widehat{p}\right]\left(\partial_{2} \varphi\right)^{2}\right\}\right|_{x_{1}=0}+\left.\operatorname{coeff} \partial_{0} \dot{H}_{N}^{+} \partial_{2} \varphi\right|_{x_{1}=0}+P
$$


где coeff $=\left(1-\widehat{\sigma}^{2}\right)\left[\partial_{1} \widehat{H}_{\tau}\right]$ (на самом деле конкретное выражение для этого коэффициента не имеет значения) и $P-$ квадратичная форма на границе, являющаяся суммой в некотором смысле младших членов, граничные интегралы от которых могут быть оценены стандартными методами (например, переходом от интеграла по границе $\partial \Omega_{t}$ к интегралу по области $\Omega_{t}$ и интегрированием по частям; см. $\left.[17 ; 18]\right)$. Первое слагаемое в правой части равенства (88) играет самую важную роль, поскольку в силу неравенства $\widehat{\Gamma}^{+}>1$ и условия Рэлея - Тейлора (63) оно после интегрирования по времени дает контроль над нормой $\left\|\partial_{2} \varphi(t)\right\|_{L^{2}(\partial \Omega)}$ (здесь нам важно только то, что положительное $\widehat{\Gamma}^{+}$«отделено» от нуля). Оценивание граничного интеграла от второго слагаемого в правой части равенства (88) является самым нетривиальным в идейном смысле местом при выводе априорной оценки (73). Для этого используется имеющийся контроль над нормой $\left\|\partial_{2} \varphi(t)\right\|_{L^{2}(\partial \Omega)}$, переход к интегралу по области, интегрирование по частям, использование неравенства Юнга и т. д. Так как для нашей задачи эти рассуждения полностью совпадают с рассуждениями для нерелятивистского случая, мы просто в очередной раз отсылаем читателя к работам [17; 18].

В итоге, как и в [17], мы получаем $L^{2}$-оценку для $\partial_{2} \dot{U}$ и $\partial_{2} \varphi$, объединяя которую с оценками $(74)$ и $(81)$, а также с $L^{2}$-оценкой для $\partial_{t} \varphi$, которая следует из последнего граничного условия в $(61)($ при $g=0)$, приходим к энергетическому неравенству (82). Это неравенство дает априорную оценку (73), а после возврата к неоднородным граничным условиям, мы получаем априорную оценку (64).

Как и в [17], доказательство существования решений линеаризованной задачи (60)-(62) проводится с помощью так называемой нехарактеристической «строго диссипативной» регуляризации этой задачи (см. подробности в [17]). Понятно, что единственность решения следует из базовой априорной оценки (64).

\section{§5. Схема доказательства теоремы 3.1}

Доказательство теоремы 3.1 полностью аналогично доказательству соответствующей теоремы для нерелятивистского случая [18]. Поэтому, отсылая читателя к работе [18], мы очень коротко обсудим схему доказательства существования решений нелинейной задачи (34)-(36). Единственность решения нелинейной задачи доказывается стандартным способом (см., например, [21]) и следует из базовой априорной оценки (64) для линеаризованной задачи.

Прежде чем доказывать существование решений, необходимо определить условия согласования начальных данных и граничных условий. За- 
тем в предположении, что начальные данные согласованы вплоть до некоторого порядка $\mu$ (см. [18]), определяется так называемое аппроксимационное решение

$$
\left(\left(U^{a+}, U^{a-}\right), \varphi^{a}\right) \in H^{\mu+1}\left(\Omega_{T}\right) \times H^{\mu+1}\left(\partial \Omega_{T}\right),
$$

которое удовлетворяет граничным условиям, начальным данным, а также условиям

$$
\left.\partial_{t}^{j} \mathbb{L}\left(U^{a \pm}, \Psi^{a \pm}\right)\right|_{t=0}=0 \quad \text { в } \Omega \text { при } j=0, \ldots, \mu-1,
$$

где $\Psi^{a \pm}=\chi\left( \pm x_{1}\right) \varphi^{a}$. Далее в рассмотрение вводятся вектор-функции

$$
f^{a \pm}:= \begin{cases}-\mathbb{L}\left(U^{a \pm}, \Psi^{a \pm}\right) & \text { при } t>0 \\ 0 & \text { при } t<0\end{cases}
$$

для которых, используя (89), можно показать, что

$$
\left\|f^{a \pm}\right\|_{H^{\mu}\left(\Omega_{T}\right)} \leq \delta_{0}(T)
$$

где константа $\delta_{0}(T) \rightarrow 0$ при $T \rightarrow 0$.

После этого в задаче (34)-(36) делается сдвиг неизвестного на аппроксимационное решение. Обозначая «сдвинутое» неизвестное так же, как и исходное неизвестное $\left(\left(U^{+}, U^{-}\right), \varphi\right)$, получаем для него задачу с нулевыми начальными данными:

$$
\begin{aligned}
& \mathcal{L}\left(U^{ \pm}, \Psi^{ \pm}\right)=f^{a \pm} \quad \text { в } \Omega_{T}, \\
& \mathcal{B}\left(U^{+}, U^{-}, \varphi\right)=0 \text { на } \partial \Omega_{T}, \\
& \left(\left(U^{+}, U^{-}\right), \varphi\right)=0 \text { при } t<0,
\end{aligned}
$$

где

$$
\begin{aligned}
\mathcal{L}\left(U^{ \pm}, \Psi^{ \pm}\right) & :=\mathbb{L}\left(U^{a \pm}+U^{ \pm}, \Psi^{a \pm}+\Psi^{ \pm}\right)-\mathbb{L}\left(U^{a \pm}, \Psi^{a \pm}\right), \\
\mathcal{B}\left(U^{+}, U^{-}, \varphi\right) & :=\mathbb{B}\left(U^{a+}+U^{+}, U^{a-}+U^{-}, \varphi^{a}+\varphi\right) .
\end{aligned}
$$

Существование решений задачи (90)-(92) доказывается с помощью подходящих итераций Нэша - Мозера. Общее описание метода Нэша - Мозера применительно к задачам типа задачи (90)-(92) можно найти в [20] (см. также ссылки внутри). Основная идея метода состоит в решении нелинейного уравнения $F(u)=0$ итерационной схемой

$$
F^{\prime}\left(S_{\theta_{n}} u_{n}\right)\left(u_{n+1}-u_{n}\right)=-F\left(u_{n}\right),
$$

где $F^{\prime}$ - линеаризация $F$, а $S_{\theta_{n}}$ - последовательность сглаживающих операторов такая, что $S_{\theta_{n}} \rightarrow I$ при $n \rightarrow \infty$. Если $S_{\theta_{n}}=I$, то эта схема совпадает с классической схемой Ньютона. Использование сглаживающих операторов на каждом шаге схемы необходимо для компенсации потери 
производных в априорных оценках линеаризованной задачи. Для задачи (90)-(92) (так же, как и для соответствующих задач в $[18 ; 21 ; 25 ; 26])$ схема Нэша - Мозера является не совсем стандартной, поскольку на каждом ее шаге необходимо строить некоторое промежуточное состояние $u_{n+1 / 2}$, удовлетворяющее тем же ограничениям, что и основное состояние (49), относительно которого проводилась линеаризация, т. е. условиям (51)-(55).

Для доказательства сходимости итераций Нэша - Мозера базовой априорной оценки (64) недостаточно. Во-первых, понятно, что нужна соответствующая оценка в старших нормах, т. е. оценка в нормах соболевских пространств $H^{s}$, где $s$ достаточно велико, чтобы по теоремам вложения можно было бы оценивать супремум-нормы через нормы в $H^{s}$. Во-вторых, для доказательства сходимости итераций Нэша - Мозера нужна более деликатная априорная оценка, которая бы учитывала не только, сколько производных правых частей линеаризованной задачи теряется по сравнению с гладкостью решений, но и то, какая гладкость требуется от коэффициентов задачи, т. е. от основного состояния (49). Следуя [18] и учитывая замечания, сделанные в предыдущем параграфе для нашего релятивистского случая, выпишем здесь такую «деликатную» априорную оценку. Априорные оценки такого типа принято называть ручными оценками (перевод с английского термина «tame estimates»).

Теорема 5.1. Пусть $T>0, s \in \mathbb{N}$ и $s \geq 3$. Предположим, что основное состояние $(\widehat{U}, \widehat{\varphi}) \in H^{s+3}\left(\Omega_{T}\right) \times H^{s+3}\left(\partial \Omega_{T}\right)$ удовлетворяет предположениям (50)-(55), условию Рэлея - Тейлора (63) и неравенству

$$
\|\widehat{U}\|_{H^{6}\left(\Omega_{T}\right)}+\|\widehat{\varphi}\|_{H^{6}\left(\partial \Omega_{T}\right)} \leq \widehat{K},
$$

где $\widehat{K}>0-$ некоторая постоянная. Пусть также

$$
(f, g) \in H^{s+1}\left(\Omega_{T}\right) \times H^{s+1}\left(\partial \Omega_{T}\right)
$$

равны нулю в прошлом. Тогда существует такая положительная постоянная $K_{0}$, которая не зависит от $s$ и $T$, и существует такая постоянная $C\left(K_{0}\right)>0$, что если $\widehat{K} \leq K_{0}$, то существует единственное решение $(\dot{U}, \varphi) \in H^{s}\left(\Omega_{T}\right) \times H^{s}\left(\partial \Omega_{T}\right)$ задачи (60)-(62). Для этого решения имеет место следующая ручная априорная оценка:

$$
\begin{aligned}
& \|\dot{U}\|_{H^{s}\left(\Omega_{T}\right)}+\|\varphi\|_{H^{s}\left(\partial \Omega_{T}\right)} \\
& \leq C\left(K_{0}\right)\left\{\begin{array}{l}
\|f\|_{H^{s+1}\left(\Omega_{T}\right)}+\|g\|_{H^{s+1}\left(\partial \Omega_{T}\right)} \\
\left.\quad+\left(\|f\|_{H^{4}\left(\Omega_{T}\right)}+\|g\|_{H^{4}\left(\partial \Omega_{T}\right)}\right)\left(\|\widehat{U}\|_{H^{s+3}\left(\Omega_{T}\right)}+\|\widehat{\varphi}\|_{H^{s+3}\left(\partial \Omega_{T}\right)}\right)\right\}
\end{array}\right.
\end{aligned}
$$

для достаточно малого времени $T$ (постоянная $C\left(K_{0}\right)$ зависит также от фиксированных констант $\bar{p}, \nu, \kappa, \epsilon$ из (51), (52), (54) и (31)). 
Ручная оценка (94) отличается от соответствующей оценки для нерелятивистского случая в [18] только тем, что, как и в базовой оценке (64), мы «теряем одну производную» для правой части $f$. Как уже отмечалось выше, это никак не влияет на окончательный результат для исходной нелинейной задачи, поскольку именно оценка вида (94) использовалась в [18] для доказательства сходимости итераций Нэша - Мозера. Вывод самой оценки (94) проводится по той же схеме, что и получение базовой априорной оценки (64), но с применением известных неравенств типа Мозера (являющихся следствием неравенства Гальярдо - Ниренберга) для оценивания коммутаторов, т. е. младших членов, возникающих при выписывании производных высших порядков от системы (60) и граничных условий (61) (cм. [18]).

На этом мы заканчиваем наши короткие комментарии по поводу доказательства теоремы 3.1 и еще раз отсылаем читателя к доказательству аналогичной теоремы в [18], где можно найти все детальные рассуждения и их технические подробности.

\section{Список литературы}

1. Блохин А. М., Дружинин И. Ю. Корректность некоторых линейных задач об устойчивости сильных разрывов в магнитной гидродинамике // Сиб. матем. журн. 1990. Т. 31, № 2. С. 3-8.

2. Блохин А. М., Трахинин Ю. Л. Устойчивость сильных разрывов в магнитной гидродинамике и электрогидродинамике. М.; Ижевск: Институт компьютерных исследований, 2004.

3. Вольперт А. И., Худяев С. И. О задачи Коши для составных систем нелинейных дифференциальных уравнений // Матем. сб. 1972. Т. 87, № 4. P. 504-528.

4. Годунов С. К. Симметрическая форма уравнений магнитной гидродинамики // Численные методы механики сплошной среды. 1972. Т. 3, № 1. С. 26-34.

5. Ландау Л. Д., Лифшиц Е. М. Электродинамика сплошных сред. М.: Наука, 1982.

6. Alinhac S. Existence d'ondes de raréfaction pour des systèmes quasilinéaires hyperboliques multidimensionnels // Comm. Partial Differential Equations. 1989. V.14, N 2. P. 173-230.

7. Anile A. M. Relativistic Fluids and Magneto-Fluids with Applications in Astrophysics and Plasma Physics. Cambridge: Cambridge Univ. Press, 1989. 
8. Anile A. M. and Pennisi S. On the mathematical structure of test relativistic magnetofluiddynamics // Ann. Inst. H. Poincaré, Phys. Theór. 1987. V. 46, N 1. P. 27-44.

9. Antón L., Miralles J. A., Martí J. M., Ibáñez J. M., Miguel A. A., and Mimica $P$. Relativistic magnetohydrodynamics: renormalized eigenvectors and full wave decomposition Riemann solver // Astrophys. J., Suppl. Ser. 2010. V. 188, N 1. P. 1-31.

10. Ebin $D$. The equations of motion of a perfect fluid with free boundary are not well-posed // Comm. Partial Differential Equations. 1987. V. 12, N 10. P. 1175-1201.

11. Freistühler H. and Trakhinin Y. Symmetrizations of RMHD equations in terms of primitive variables and their application to relativistic currentvortex sheets // Classical Quantum Gravity. 2013. V.30, N 8, ID 085012.

12. Goedbloed J. P., Keppens R., and Poedts S. Advanced Magnetohydrodynamics: with Applications to Laboratory and Astrophysical Plasmas. Cambridge, UK: Cambridge Univ. Press, 2010.

13. Guo Y. and Tice I. Compressible, inviscid Rayleigh-Taylor instability // Indiana Univ. Math. J. 2011. V.60, N 2. P.677-711.

14. Joseph D. D. and Saut J.-C. Short-wave instabilities and ill-posed initialvalue problems // Theor. Comp. Fluid Dyn. 1990. V.1, N 4. P. 191-227.

15. Kato T. The Cauchy problem for quasi-linear symmetric hyperbolic systems // Arch. Rational Mech. Anal. 1975. V.58, N 3. P. 181-205.

16. Lichnerowicz A. Relativistic Hydrodynamics and Magnetohydrodynamics / Lectures on the Existence of Solutions. New York; Amsterdam: Benjamin, Inc., 1967.

17. Morando A., Trakhinin Y., and Trebeschi P. Well-posedness of the linearized problem for MHD contact discontinuities // J. Differential Equations. 2015. V. 258, N 7. P. 2531-2571.

18. Morando A., Trakhinin Y., and Trebeschi P. Local existence of MHD contact discontinuities // Arch. Rational Mech. Anal. 2018. V.228, N7. P. 691-742.

19. Ruggeri T. and Strumia A. Convex covariant entropy density, symmetric conservative form, and shock waves in relativistic magnetohydrodynamics // J. Math. Phys. 1981. V.22, N 8. P. 1824-1827.

20. Secchi P. On the Nash-Moser iteration technique // Recent Developments of Mathematical Fluid Mechanics / Series: Advances in Mathematical Fluid Mechanics. Eds. H. Amann et al. Basel: Springer, 2016. P. 443-457.

21. Secchi P. and Trakhinin Y. Well-posedness of the plasma-vacuum interface problem // Nonlinearity. 2014. V.27, N1. P. 105-169. 
22. Trakhinin $Y$. On stability of shock waves in relativistic magnetohydrodynamics // Quart. Appl. Math. 2001. V.59, N 1. P. 25-45.

23. Trakhinin $Y$. A complete 2D stability analysis of fast MHD shocks in an ideal gas // Comm. Math. Phys. 2003. V.236, N 1. P. 65-92.

24. Trakhinin Y. On existence of compressible current-vortex sheets: Variable coefficients linear analysis // Arch. Rational Mech. Anal. 2005. V. 177, N 3. P. 331-366.

25. Trakhinin $Y$. The existence of current-vortex sheets in ideal compressible magnetohydrodynamics // Arch. Rational Mech. Anal. 2009. V.191, N 2. P. 245-310.

26. Trakhinin Y. Local existence for the free boundary problem for nonrelativistic and relativistic compressible Euler equations with a vacuum boundary condition // Comm. Pure Appl. Math. 2009. V.62, N 11. P. 1551-1594.

27. Trakhinin $Y$. On the well-posedness of a linearized plasma-vacuum interface problem in ideal compressible MHD // J. Differential Equations. 2010. V. 249, N 10. P. 2577-2599.

28. Trakhinin Y. On well-posedness of the plasma-vacuum interface problem: The case of non-elliptic interface symbol // Comm. Pure Appl. Anal. 2016. V. 15, N 4. P. 1371-1399.

Трахинин Юрий Леонидович

Институт математики им. С. Л. Соболева СО РАН, просп. Академика Коптюга, 4, Новосибирск, 630090 РОССИЯ.

Новосибирский гос. университет, ул. Пирогова, 2,

Новосибирск, 630090 РОССИЯ.

E-mail: trakhin@math.nsc.ru
Поступила в редакцию 26 октября 2018 г.

Получена после доработки 26 октября 2018 г.

Принята к публикации 27 февраля 2019 г. 